\title{
Checklist of the vascular flora of Wielkopolska (Poland): casual alien species
}

\author{
Bogdan Jackowiak*, Zbigniew Celka, Julian Chmiel, Karol Latowski \\ \& Waldemar Żukowski
}

Department of Plant Taxonomy, Faculty of Biology, Adam Mickiewicz University, Umultowska 89, 61-614 Poznań, Poland

* corresponding author (e-mail: bogjack@amu.edu.pl)

\begin{abstract}
The list of alien vascular plant species only temporarily occurring in Wielkopolska refers to the previously published list of native and permanently established plants. Together, these two lists document the vascular flora of this region at the beginning of the 21 st century. The current list, like the previous one, is a result of critical analysis of both contemporary and historical data, collected since the beginning of the 19th century. All information accessible in herbarium collections, publications and unpublished materials was used. A critical analysis was conducted at the taxonomic, nomenclatural, chorological and habitat levels, based on the verification of negative information not supported by sufficient arguments. The list is presented in an alphabetical order. Information on each species includes: family affinity, life form, geographic and historic status. In cases particularly disputable, the standard characteristic of a species was supplemented with an additional commentary.
\end{abstract}

Key words: regional biodiversity assessment, flora, vascular plants, alien species, synanthropisation, Great Poland, Central Europe

\section{Introduction}

The appearance of alien species in the flora has been a subject of intensive research for a long time. This phenomenon was noticed already in the mid-nineteenth century by De Candolle (1855), who introduced the term of an adventive plant to literature. The classification regarding the time of introduction and the stability of occurrence of alien species in a new region was of key importance for the development of research in this area (Thellung 1918/1919). With reference to the concept of A. Thellung, many variants of the classification of alien plants and definitions of particular groups of anthropophytes were created in the twentieth century (e.g., Holub \& Jirásek 1967; Kornaś 1968, 1977, 1981; Schroeder 1969; Jackowiak 1990; Protopopova 1991). Finally, an attempt was made to put in order the classification system and definitions (Pyšek et al. 2004), because research in this field is developing very dynamically.

In many countries and regions, the appearance of alien species is carefully analyzed and evaluated in terms of biodiversity changes and the invasion process. Alien plant species were commonly recognized in the floras of many countries (Crawley et al. 1996; Celesti-Grapow et al. 2009; Pyšek et al. 2012; Protopopova \& Shevera 2014; Elvisto et al. 2016). This phenomenon has also been summed up on a European scale (Lambdon et al. 2008). However, it is still necessary to monitor this phenomenon both on a regional and local scale, and to pay even greater attention to casual alien species.

The subject of this paper are alien species occurring accidentally in Wielkopolska (western part of Poland) over the whole period of geobotanical research conducted in this region. The aim of the study was to evaluate the changes in regional flora resulting from the presence of accidentally introduced alien species. The checklist of casual alien species supplements the list of native and permanently established species of Wielkopolska (Jackowiak et al. 2013b).

The observations of alien plant species appearing in Wielkopolska have a long history (Jackowiak et al. 2013a). The first scientific work on this region was 
published at the end of the 19th century: "Immigration of some plants in the city of Poznań after 1850 year ..." (Pfuhl 1896). In the first half of the twentieth century, two works are primarily noteworthy: $(i)$ "The newcomers and waifs in the vegetation of Poznań" (Szulczewski 1931) and (ii) "Geographical analysis of the synanthropic flora of the city of Poznan" (Krawiecowa 1951). Both in the second half of the twentieth century and presently, foreign plant species have been as standard included in local floristic studies. Particular attention has been paid to alien plant species in geobotanical monographs devoted to anthropogenic changes of the flora of Poznan (Jackowiak 1990, 1993), Gniezno Lake District (Chmiel 1993) and archeological objects (Celka 1999, 2004). A lot of data from Wielkopolska has been included in works concerning the entire country (Rostański \& Sowa 1986/1987; Tokarska-Guzik 2005; Urbisz 2012).

\section{Material and methods}

\subsection{Limits of the research area and material}

The area of study almost entirely coincides with the Wielkopolska-Kujawy Lowland, thus, it corresponds to a second-level unit in the geobotanical division of Poland (Szafer 1972). The research area also included fragments of two other geobotanical units: the Northern Marginal Plateau Region and TrzebnickoOstrzeszowskie Hills.
Similarly as in our previous publications (e.g., Żukowski \& Jackowiak 1995; Jackowiak et al. 2013a, 2013b), Wielkopolska is defined in this study within broad limits, covering both Kujawy (in the east) and Ziemia Lubuska (Central Nadodrze) in the west (Fig. 1). In total, the area of research covered about 50 thousand $\mathrm{km}^{2}$.

The list of plant species found in the Wielkopolska region includes data from all available literature sources, as well as materials collected in the herbarium of Adam Mickiewicz University (POZ), and verified own and other authors' data not yet published. The main list of sources is included in the work on the history of floristic studies in Wielkopolska (Jackowiak et al. 2013a). Currently, it has been supplemented with publications that appeared in 2013-2017.

\subsection{Taxonomy, nomenclature and basis for species classification}

The alphabetical list of alien species not established in the vascular flora of Wielkopolska includes: name of the species and its author, in some cases also synonyms, family name, life form, information on native range, classification according to the source of introduction, and data regarding the observation period Additional notes have been added to several dozen of species.

The taxonomy, nomenclature and biological form of species, as well as the region of origin, were determined primarily on the basis of the flora of Rothmaler et al. (2005), Jäger et al. (2008) and the Plant List (2013). In

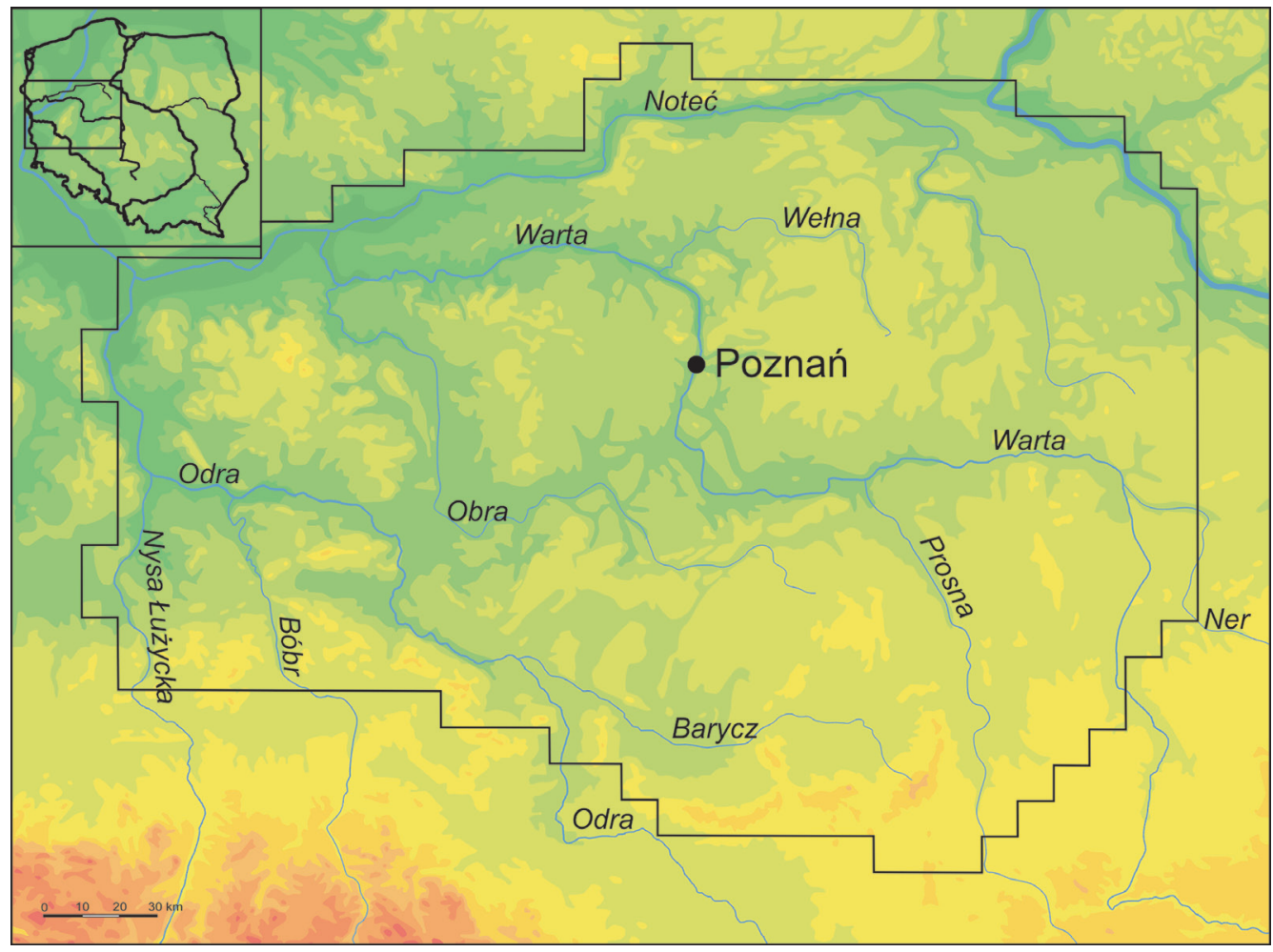

Fig. 1. Location and borders of the Wielkopolska Region (according to Jackowiak et al. 2007) 
general, species names are given, only in justified cases, subspecies names were used (e.g. Lathyrus japonicus Willd. subsp. maritimus (L.) P. W. Ball). The list also includes hybrid species (e.g.,. Mentha ×piperita L.).

The following categories of plants are included in the classification of biological forms: (1) annual plants, including summer annual plants that sprout, flower, produce seeds, and die during the warmer months of the year, as well as annual plants that germinate in autumn or winter, live through the winter, then bloom in winter or spring; (2) biennial plants that take two years to complete their biological lifecycle. In the first year, the plant grows leaves, stems, and roots (vegetative structures), then it enters a period of dormancy over the colder months. Usually, the stem remains very short and leaves are low to the ground, forming a rosette; (3) perennial, monocarpic plants that live more than two years, but they die after the first fruiting; (4) perennial, polycarpic plants that live, bloom and bear fruit for more than two years; (5) dwarf shrubs and sub-shrubs; (6) shrubs and trees.

In terms of the source of the introduction, casual alien species (diaphytes sensu Kornaś 1981) were divided into two subgroups: ephemerophytes and ergasiophygophytes (Thellung 1918/19). The first sub-group includes species not cultivated in Wielkopolska and neighboring regions, but brought from other areas ("occasional escapees" sensu Munz 1968, and "ephemeral taxa" sensu Elven \& Elvebakk 1996). They correspond to the term "adventive" introduced by De Candolle (1855), later often used both in this and a much broader sense (Muhlenbach 1979; Burda 1991; Provost 1998). Only for species belonging to this subgroup, the region of origin was given. The second subgroup includes casual alien species spreading from cultivation ("waifs" sensu Hickman 1993).

Important information, especially in the case of ephemeral species, is their observation date. Taking into account the history of floristic research in Wielkopolska (Jackowiak et al. 2013a), data on the appearance of species were divided into four periods: (1) until 1901, (2) in the years 1901-1951; (3) in the years 1952-2001; (4) after 2001. It was also indicated whether the species was observed once or repeatedly.

\section{Results}

\subsection{Systematic structure of casual alien plant flora}

The list of alien plants appearing ephemerally in Wielkopolska from the beginning of the 19th century to the present includes 481 species (Appendices 1-2). They represent 297 genera and 80 families.

The distribution of species within families is very diverse. Close to $60 \%$ of the flora of casual alien plants consists of species belonging to 10 families that are the most numerous in Wielkopolska (Table 1). On the other hand, worth of noting is the high number of families represented by a single species ( 33 families), and two species (14 families).

Table 1. Species diversity of families represented in the flora of casual alien plants of Wielkopolska

\begin{tabular}{|c|c|c|c|c|}
\hline Family name & $\begin{array}{l}\text { Number } \\
\text { of species } \\
\text { in the family }\end{array}$ & $\begin{array}{l}\text { Number } \\
\text { of families }\end{array}$ & $\begin{array}{l}\text { Total } \\
\text { number } \\
\text { of species }\end{array}$ & $\%$ \\
\hline Asteraceae & 62 & 1 & 62 & 12.9 \\
\hline Poaceae & 46 & 1 & 46 & 9.6 \\
\hline Brassicaceae & 37 & 1 & 37 & 7.7 \\
\hline Rosaceae & 34 & 1 & 34 & 7.1 \\
\hline Fabaceae & 30 & 1 & 30 & 6.2 \\
\hline Liliaceae & 21 & 1 & 21 & 4.4 \\
\hline Lamiaceae & 19 & 1 & 19 & 4.0 \\
\hline Caryophyllaceae & 15 & 1 & 15 & 3.1 \\
\hline Apiaceae & 14 & 1 & 14 & 2.9 \\
\hline Chenopodiaceae, Solanaceae & 12 & 2 & 24 & 5.0 \\
\hline Onagraceae, Polygonaceae & 9 & 2 & 18 & 3.7 \\
\hline Malvaceae & 8 & 1 & 8 & 1.7 \\
\hline Boraginaceae, Papaveraceae, Pinaceae & 7 & 3 & 21 & 4.4 \\
\hline Amaranthaceae, Cucurbitaceae, Ranunculaceae, Salicaceae & 6 & 4 & 24 & 5.0 \\
\hline Campanulaceae, Cuscutaceae, Scrophulariaceae & 5 & 3 & 15 & 3.1 \\
\hline Caprifoliaceae, Euphorbiaceae, Linaceae, Resedaceae, Rubiaceae & 4 & 5 & 20 & 4.2 \\
\hline Convolvulaceae, Fumariaceae, Plantaginaceae, Violaceae & 3 & 4 & 12 & 2.5 \\
\hline see Appendix 1 and 2 & 2 & 14 & 28 & 5.8 \\
\hline see Appendix 1 and 2 & 1 & 33 & 33 & 6.9 \\
\hline Total & & 80 & 481 & 100.0 \\
\hline
\end{tabular}


Table 2. Species diversity of genera represented in the flora of casual alien plants of Wielkopolska

\begin{tabular}{lrrrr}
\hline Names of genera & $\begin{array}{l}\text { Number } \\
\text { of species } \\
\text { in genus }\end{array}$ & $\begin{array}{c}\text { Number } \\
\text { of } \\
\text { genera }\end{array}$ & $\begin{array}{c}\text { Total } \\
\text { number } \\
\text { of species }\end{array}$ & $\%$ \\
\hline Allium & 9 & 1 & 9 & 1.9 \\
Oenothera & 8 & 1 & 8 & 1.7 \\
Bromus, Chenopodium, Rosa & 7 & 3 & 21 & 4.4 \\
Amaranthus, Artemisia, Centaurea, Spiraea, Vicia & 6 & 5 & 30 & 6.2 \\
Cuscuta, Lathyrus, Silene & 5 & 3 & 15 & 3.1 \\
Linum, Pinus, Prunus, Rumex, Salix, Solanum & 4 & 6 & 24 & 5.0 \\
$\quad$ see Appendix 1 and 2 & 3 & 21 & 63 & 13.1 \\
\multicolumn{1}{c}{ see Appendix 1 and 2 } & 2 & 54 & 108 & 22.5 \\
$\quad$ see Appendix & 1 & 203 & 203 & 42.2 \\
$\quad$ Total & & $\mathbf{2 9 7}$ & $\mathbf{4 8 1}$ & $\mathbf{1 0 0 . 0}$ \\
\hline
\end{tabular}

The distribution of species in genera is very characteristic. The most numerous group consists of genera represented by single or two species. These genera constitute together nearly $65 \%$ of the analyzed flora (Table 2).

\subsection{Biological spectrum of casual alien plant flora}

In the flora of casual alien plants of Wielkopolska dominate annual species, whose share exceeds $42 \%$ (Fig. 2). A significant share have also perennial and polycarpic herbaceous plants, followed by trees and shrubs. On the other hand, biennial plants and shrubs and semi-shrubs are of minor importance. The group "other" includes species that may occur in various biological forms, but the lack of information from the region prevents their unambiguous determination.

\subsection{Geographical origin, sources of dispersion and} the frequency of records of casual alien species

Among casual alien species, escapees from crops predominate, constituting $60.3 \%$ of the analyzed flora (290 species). Thus, the share of foreign plants, introduced directly from other geographical areas, is $39.7 \%$ (191 species). In many cases, it was not easy to classify a species into a proper group, due to the limited knowledge of crops grown within the contemporary borders of Wielkopolska, especially in the 19th and the first half of the 20th century. For this reason, the

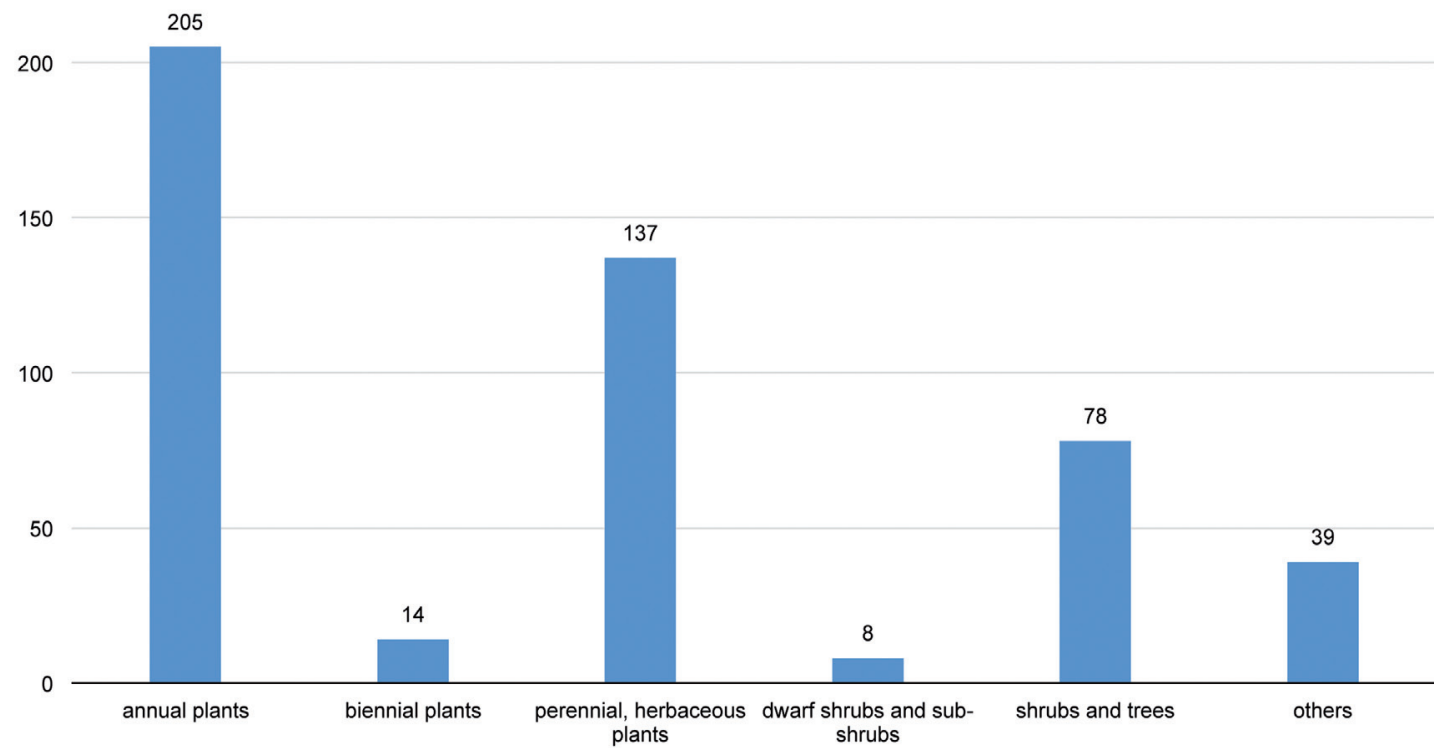

Fig. 2. Share of biological forms in the flora of casual alien plants of Wielkopolska 
Table 3. The number of ephemerophytes representing different continents and range groups in the flora of Wielkopolska

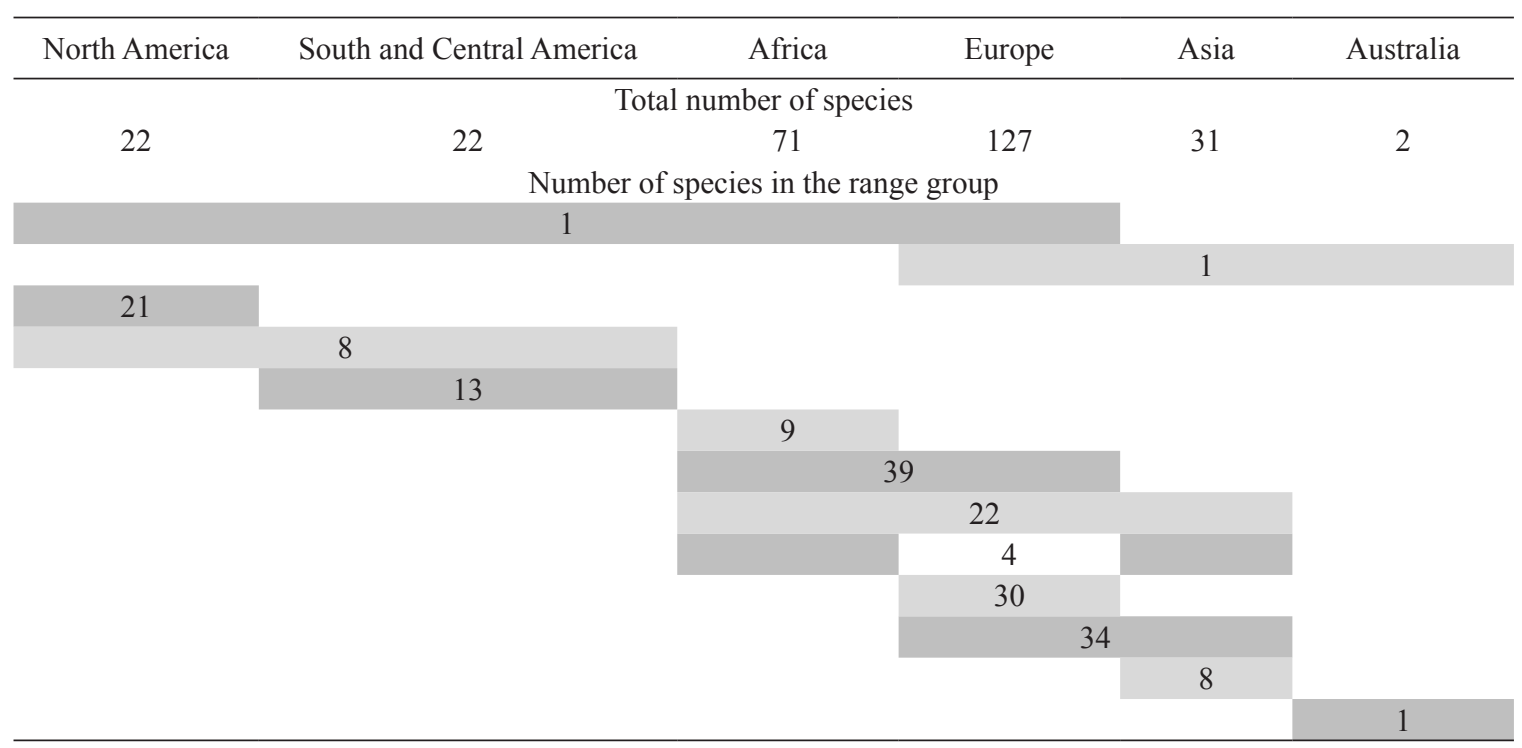

status of certain species may prove to be controversial.

The frequency of records of casual alien species is very low. Over $70 \%$ of them were observed only once (338 species), while about 30\% were recorded from two to several times (136 species). It is worth noting that there is a significant difference between the two subgroups of casual alien species. Over $81 \%$ of ephemerophytes (155 species) were recorded only once. However, ergasiophygophytes are significantly more frequently observed, of which about $63.1 \%$ of species have been observed only once (183 species).

The phytogeographic analysis of ephemerophytes shows that in the flora of Wielkopolska, plants from all continents are represented (Table 3 ). The most numerous group consists of alien species whose native ranges cover Europe (66.5\%). They include species with very different primary ranges covering: only Europe (30 species), southern and southwestern Europe and northern Africa (39 species), Eurasia (34 species) and finally Europe, Asia and North Africa (22 species). The second place is occupied by a group of species covering different parts of the African continent (39.3\%). It is worth noting here that next to the taxa with a wider intercontinental range, there are also plants whose native range is limited to Africa (9 species). The third place is taken by a group of plants distributed in Asia (18.3\%), including 8 species found naturally only on this continent. The participation of elements representing North America and South America, including Central America is the same (11.5\% each). Among the ephemerophytes, one species originating from Australia was recorded.

\section{Conclusion}

The full list of vascular plants found in the Wielkopolska region during nearly 200 years includes more than 2,230 species (Jackowiak et al. 2013b). A significant part of the flora is composed of non-established species (481). This means that every fifth species $(21.6 \%)$ occurs only temporarily. As the history of the invasion of many plant species shows, it is possible that at least some of casual alien taxa may become permanently established in the future. Therefore, regular monitoring of casual alien species is required. This applies to both escapees from crops, which prevail on the checklist of flora of Wielkopolska, and ephemerophytes that spread regardless of human activity. 


\section{References}

Antkowiak W., Czarna A. \& Wawrzyniak M. 2008. Pyrus $\times$ myloslavensis (P. communis L. $\times$ P. salicifolia Pall.) - a new spontaneouns pear hybrid. Dendrobiology 60: $45-49$

Burda R. I. 1991. Antropogennaia Transformacia Flory. Naukova Dumka, Kiev.

Celesti-Grapow L., Alessandrini, A., Arrigoni P. V., Assini S., Banfi E., Barni E., Bovio M., Brundu G., Cagiotti M. R., Camarda I., Carli E.. Conti F., Del Guacchio E., Domina G., Fascetti S., Galasso G., Gubellini L., La Valva V., Lucchese F., Marchiori S., Mazzola P., Peccenini S., Poldini L., Pretto F., Prosser F., Siniscalco C., Villani M. C., Viegi L., Wilhalm T. $\&$ BLASI C. 2009. Inventory of the non-native flora of Italy. Plant Biosystems 143 (2): 386-430.

CELKa Z. 1999. Rośliny naczyniowe grodzisk Wielkopolski. Prace Zakładu Taksonomii Roślin UAM w Poznaniu 9: 1-159. Bogucki Wyd. Nauk., Poznań.

Celka Z. 2004. Distribution Atlas of Vascular Plants on the Earthworks of Wielkopolska. Publications of the Department of Plant Taxonomy of Adam Mickiewicz University in Poznań 13: 1-447. Bogucki Wyd. Nauk., Poznań.

Chmiel J. 1993. Flora roślin naczyniowych wschodniej części Pojezierza Gnieźnieńskiego i jej antropogeniczne przeobrażenia w wieku XIX i XX, cz. 1 i 2. Prace Zakładu Taksonomii Roślin UAM w Poznaniu 1; 1 : 1-202, 2: 1-212. Wyd. Sorus, Poznań.

Chmiel J. 2006. Zróżnicowanie przestrzenne flory jako podstawa ochrony przyrody w krajobrazie rolniczym. Prace Zakładu Taksonomii Roślin UAM w Poznaniu 14: 1-250. Bogucki Wyd. Nauk., Poznań.

Crawley M. J., Harvey P. H. \& Purvis A. 1996. Comparative ecology of the native and alien floras of the British Isles. Phil. Trans. R. Soc. Lond. B 351(1345): 12511259. DOI: $10.1098 /$ rstb. 1996.0108

Czarna A. 2003. Cicerbita macrophylla (Willd.) Wallr. wWielkopolsce. Rocz. AR Pozn. 354, ser. Bot. 6: 15-16.

Czarna A. 2005. Vascular flora of the railwey station in Jarocin (Western Poland). Rocz. AR Pozn. 373, ser. Bot.-Stec. 9: 39-46.

CzARnA A. 2007. Current flora of herbaceous plants in castle park in Gołuchów (Wielkopolska region, Poland). Rocz. AR Pozn. 384, ser. Bot.-Stec. 11: 35-47.

Czarna A. 2009. Rośliny naczyniowe środkowej Wielkopolski. 184 pp. Wyd. Uniwersytetu Przyrodniczego w Poznaniu.

Czarna A. \& Mielcarski Cz. 2004. Salvia glutinosa L. na terenie miasta Poznania. Rocz. AR Pozn. CCCLXIII, ser. Bot. 7: 47-51.

Danielewicz W. \& Maliński T. 1994. Obce gatunki drzew i krzewów w Wielkopolskim Parku Narodowym. Przegl. Przyrodn. 5(3/4): 181-190.

DANIELEWICZ W. \& MALIŃSKI T. 1995. Materiały do znajomości dendroflory Wielkopolskiego Parku Narodowego. Morena 3: 7-27.

Danielewicz W. \& Maliński T. 1997. Drzewa i krzewy obcego pochodzenia w lasach Wielkopolskiego Parku Narodowego. Rocz. Dendrol. 45: 65-81
De Candolle A. P. 1855. Géographie Botanique Raisonné, vol. 2. V. Masson, Paris.

Dolatowski J. \& Seneta W. 2008. Dendrologia. 544 pp. Wyd. Nauk. PWN, Warszawa.

Elven R. \& Elvebakk A. 1996. Part 1. Vascular plants. In: A. Elvebakk \& P. Prestrud (eds.). A Catalogue of Svalbard Plants, Fungi, Algae, and Cyanobacteria. Norsk Polarinstitut Skrifter 198: 9-55.

Elvisto T., Margus Pensa M. \& Paluoja E. 2016. Indigenous and alien vascular plant species in a northern European urban setting (Tallinn, Estonia). Plant Geography. Proceedings of the Estonian Academy of Sciences 64 (4): 431-441.

GąBKa M. \& Owsianny P. M. 2009. First records of the Hygrophila polysperma (Roxb.) T. Anderson (Acanthaceae) in Poland. Roczniki Akademii Rolniczej w Poznaniu. Botanika-Steciana 13: 9-14.

Golenia A. \& Bayerowa H. 1956. Kanianka polna (Cuscuta arvensis Beyr. var. calycina Engelm.; C. pentagona, calycina Engelm.) i jej nowe stanowiska w Polsce. Fragm. Flor. Geobot. 2(2): 5-9.

Hickman J. C. (ed.). 1993. The Jepson Manual. Univ. California Press, Berkeley.

Holub J. \& JirÁseK V. 1967. Zur Vereinheitlichung der Terminologie in der Phytogeographie. Folia Geobot. Phytotax. 2: 69-113.

JACKOWIAK B. 1990. Antropogeniczne przemiany flory roślin naczyniowych Poznania. Wyd. Nauk. UAM, seria Biologia, 42, 232 pp. Poznań.

JACKOWIAK B. 1993. Atlas of distribution of vascular plants in Poznań. Publications of the Department of Plant Taxonomy of the Adam Mickiewicz University in Poznań 3: 1-409.

Jackowiak B., Celka Z., Chmiel J., Latowski K. \& ŻUKOwsKi W. 2007. Red list of vascular flora of Wielkopolska (Poland). Biodiv. Res. Conserv. 5-8: 95-127.

Jackowiak B., Celka Z., Chmiel J., Latowski K. \& Żukowski W. 2013a. The history of research on the vascular flora of Wielkopolska (Poland). Biodiv. Res. Conserv. 31: 1-8.

Jackowiak B., Celka Z., Chmiel J., Latowski K. \& Żukowski W. 2013b. Checklist of the vascular flora of Wielkopolska (Poland): native species and naturalized alien species. Biodiv. Res. Conserv. 31: 9-96.

Jäger E. J., Ebel F., Hanelt P. \& Müller G. K. (eds.). 2008. Exkursionsflora von Deutschland. Band 5, Krautige Zier- und Nutzpflanzen. 880 pp. Spektrum Akademischer Verlag, Springer-Verlag Berlin, Heidelberg.

Kaźmierczakowa R. 2014a. CR Artemisia pontica L. In: R. Kaźmierczakowa, K. Zarzycki \& Z. Mirek (eds.). Polska Czerwona Księga Roślin. Paprotniki i rośliny kwiatowe, wyd. 3, pp. 521-522. PAN, Instytut Ochrony Przyrody, Kraków.

Kaźmierczakowa R. 2014b. VU Reseda phyteuma L. In: R. Kaźmierczakowa, K. Zarzycki \& Z. Mirek (eds.). Polska Czerwona Księga Roślin. Paprotniki i rośliny kwiatowe, wyd. 3, pp. 234-236. PAN, Instytut Ochrony Przyrody, Kraków. 
KoRnAś J. 1968. Geograficzno-historyczna klasyfikacja roślin synantropijnych. Mater. Zakł. Fitosoc. Stos. U.W. 25: 33-41.

Kornaś J. 1977. Analiza flor synantropijnych. Wiad. Bot. 21(2): 85-91.

KoRnAŚ J. 1981. Oddziaływanie człowieka na florę: mechanizmy i konsekwencje. Wiad. Bot. 25(3): 165-182.

Krawiecowa A. 1951. Analiza geograficzna flory synantropijnej miasta Poznania. PTPN, Prace Kom. Biol. 13(1): 1-131.

LADEMANN O. 1937. Adventivpflanzen der östlichen Niederlausitz. Verh. Bot. Ver. Prov. Brandenburg 77: 1-24.

Lambdon P. W., Pyšek P., Basnou C., Hejda M., Arianoutsou M., Essl F., Jarošík V., Pergl J., Winter M., Anastasiu P., Andriopoulos P., Bazos I., Brundu G., CelestiGrapow L., Chassot P., Delipetrou P., Josefsson M., Kark S., Klotz S., Kokkoris Y., Kühn I., Marchante H., Perglová I., Pino J., Vilà M., Zikos A., Roy D. \& Hulme P. E. 2008. Alien flora of Europe: species diversity, temporal trends, geographical patterns and research needs. Preslia 80(2): 101-149.

LATOWSKi K. 1981. Dalsze materiały florystyczne z terenów kolejowych Wielkopolski. Bad. Fizjogr. Pol. Zach., seria B-Botanika 32: 207-211.

Latowski K. 2009. Nowe i osobliwe antropofity we florze naczyniowej Wielkopolskiego Parku Narodowego. Cz. I. Szkarlatka jagodowa [Phytolacca acinosa Roxburgh]. Morena 14: 215-222.

Meusel H., Jäger E., Rauschert S. \& Weinert E. 1978. Vergleichende Chorologie der zentraleuropäischen Flora. II. Text xi+418 pp., Karten pp. 259-421. Gustav Fischer Verlag, Jena.

Mirek Z., Piękoś-Mirkowa H., Zając A. \& Zając M. 2002. Flowering plants and pteridophytes of Poland. A checklist. In: Z. MireK (ed.). Biodiversity of Poland, 1, 442 pp. W. Szafer Institute of Botany, Polish Academy of Sciences, Kraków.

Munlenbach V. 1979. Contributions to the synanthropic (adventive) flora of the railroads in St. Louis, Missouri, U.S.A. Ann. Missouri Bot. Garden 66: 1-108.

Munz P. A. 1968. A California Flora and Supplement. Univ. California Press, Berkeley.

Oklejewicz K., Wolanin M. \& Wolanin M. N. 2015. Czerwona księga roślin województwa podkarpackiego. Zagrożone gatunki roślin. Zbiorowiska roślinne. 299 pp. Stowarzyszenie na Rzecz Rozwoju i Promocji Podkarpacia "Pro Carpathia", Rzeszów.

Pampuch A. 1840. Flora Tremesnensis. 70 pp. Trzemeszno.

Pfunl F. 1896. Die bisher in der Provinz Posen nachgewissenen Gefässpflanzen. Zeitschr. Botan. Abteil. Naturwiss. Verein. 3(1): 1-70.

Protopopova V. V. 1991. The Synanthropic Flora of Ukraine and its Development. 204 pp. Naukova Dumka Press, Kiev.

Protopopova V. V \& Shevera M. V. 2014. Ergasiophytes of the Ukrainian flora. Biodiv. Res. Conserv. 35: 31-46.

Provost M. 1998. Flore Vasculaire de Basse-Normandie, vol. 2. Université de Caen, Caen.

Purcel A. 2007. Obce gatunki drzew i krzewów na terenie Wielkopolskiego Parku Narodowego - ich udział i rola w biocenozach Parku. Ph. D. Thesis, Katedra
Przyrodniczych Podstaw Leśnictwa Akademia Rolnicza im. Augusta Cieszkowskiego w Poznaniu, Poznań.

Purcel A. 2009. Obce gatunki drzew i krzewów w Wielkopolskim Parku Narodowym - ich występowanie i rola w biocenozach parku. Morena 14: 35-191.

Pyšek P., Danihelka J., SÁdlo J., Chrtek J. JR., Chytrý M., Jarošík V., Kaplan Z. \& Krahulec F. 2012. Catalogue of alien plants of the Czech Republic (2nd edition): checklist update, taxonomic diversity and invasion patterns. Preslia 84(2): 155-255.

Pyšek P., Richardson D. M., Rejmánek M., Webster G. L., Williamson M. \& Kirschner J. 2004. Alien plants in checklists and floras: towards better communication between taxonomists and ecologists. Taxon 53(1): 131-143.

Ritschl G. 1850. Flora des Großherzogthums Posen, im Auftrage des naturhistorischen Vereins zu Posen. 291 pp. Druck und Verlag von E. S. Mittler und Sohn, Berlin.

Rothmaler W., JÄger E. J. \& Werner K. 2005. Exkursionsflora von Deutschland. Band 4, Gefäßpflanzen: Kritischer Band. 980 pp. Spektrum Akademischer Verlag, Elsevier Gmbh, München.

Rostański K. \& Sowa R. 1986/1987. Alfabetyczny wykaz efemerofitów Polski. Fragm. Flor. Geobot. 31-32 (1-2): 151-205.

Schroeder F. G. 1969. Zur Klassifizierung der Anthropochoren. Vegetatio 16: 225-238.

Sotek Z. 2014. CR Plantago coronopus L. In: R. KAźMiercZakowa, K. Zarzycki \& Z. Mirek (eds.). Polska Czerwona Księga Roślin. Paprotniki i rośliny kwiatowe, wyd. 3, pp. 485-487. PAN, Instytut Ochrony Przyrody, Kraków.

SzulczewsKi J. W. 1931. Przybysze i przybłędy w roślinności Poznania. PTPN, Prace Kom. Mat.-Przyr., seria B, 5: 59-74.

SZULCZEWSKi J. W. 1951. Wykaz roślin naczyniowych w Wielkopolsce dotąd stwierdzonych. PTPN, Prace Kom. Biol. 12(6): 1-128.

The Plant List 2013. http://www.theplantlist.org/

Thellung A. 1918/1919. Zur Terminologie der Adventiv- und Ruderalfloristik. Allg. Bot. Zeitschr. 24/25: 36-42.

TokARskA-GuZIK B. 2005. The Establishment and Spread of Alien Plant Species (Kenophytes) in the Flora of Poland. Prace naukowe Uniw. Śląskiego w Katowicach 2372: 1-192.

URBISZ A. 2012. Occurrence of temporarily-introduced alien plant species (ephemerophytes) in Poland - scale and assessment of the phenomenon. Prace naukowe Uniw. Śląskiego w Katowicach 2897: 1-200.

ZAJĄC A. 1979. Pochodzenie archeofitów występujących w Polsce. Rozpr. habil. Uniw. Jagiell, 29: 1-213. Druk UJ w Krakowie.

ZajĄC A. \& ZajĄC M. (eds.). 2001. Distribution Atlas of Vascular Plants in Poland. xii+714 pp. Edited by Laboratory of Computer Chorology, Institute of Botany, Jagiellonian University, Cracow.

ZAJĄC M., ZAJAcC A. \& ToKARSKA-GuZIK B. 2009. Extinct and endangered archaeophytes and the dynamics of their diversity in Poland. Biodiv. Res. Conserv. 13: 17-24. 
ZIELIŃSKI J. 2016. Różowokwiatowe mieszańce robinii (Robinia L., Fabaceae) w uprawie. Propozycja utworzenia grup kultywarów: Ambigua i Margarettae. Rocznik Polskiego Towarzystwa Dendrologicznego 64: 21-33.

Zieliński J., Biel G., Danielewicz W., Tomaszewski D. \& GAWLAK M. 2015. Różowokwiatowe robinie (Robinia L., Fabaceae) dziczejące w Polsce. Rocznik Polskiego Towarzystwa Dendrologicznego 63: 9-33.

ŻuKowski W. \& JACKоwiAK B. 1995. List of endangered and threatened vascular plants in Western Pomerania and Wielkopolska (Great Poland). In: W. ŻuKowsKi \& B. JACKOWIAK (eds.). Endangered and threatened vascular plants of Western Pomerania and Wielkopolska.
Publications of the Department of Plant Taxonomy of the Adam Mickiewicz University in Poznań 3: 9-96. Bogucki Wyd. Nauk., Poznań.

ŻuKowsKI W. 1959. Nowe i rzadkie gatunki synantropijne dla flory miasta Poznania. Przyr. Polski Zach. 3: 151-153. Żukowski W., Latowski K., Jackowiak B. \& Chmiel J. 1995. Rośliny naczyniowe Wielkopolskiego Parku Narodowego. Prace Zakładu Taksonomii Roślin UAM w Poznaniu 4: 1-229. Bogucki Wyd. Nauk. Poznań.

Żukowski W. \& Piaszyk M. 1971. Rozmieszczenie gatunków z rodzaju Artemisia L. w Polsce. Bad. Fizjogr. Pol. Zach. 24: 107-129. 
Appendix 1. Checklist of casual alien species of Wielkopolska - ephemerophytes

\begin{tabular}{|c|c|c|c|c|}
\hline Name of species & Name of family & $\mathbf{B F}$ & OP & Native range \\
\hline Abutilon theophrasti Med. & Malvaceae & A & $4 \mathrm{~b}$ & Asia (N China) \\
\hline Achillea nobilis L. & Asteraceae & $\mathrm{P}$ & $2 \mathrm{a}$ & Europe S, C; Asia W \\
\hline Acroptilon repens (L.) DC. & Asteraceae & $\mathrm{P}$ & $3 b$ & Asia W, C \\
\hline Aegilops cylindrica Host & Poaceae & A & $3 b, 4 b$ & Europe S, Russia \\
\hline $\begin{array}{l}\text { Aegilops speltoides Tausch subsp. } \\
\text { ligustica (Savign.) Zhuk. } \\
\text { [= Aegilops ligustica Asch. \& } \\
\text { Graebn.] }\end{array}$ & Poaceae & A & $2 \mathrm{a}$ & Medit \\
\hline Amaranthus deflexus L. & Amaranthaceae & $\mathrm{AP}$ & $4 a ?$ & S America \\
\hline Amaranthus dinteri Schinz & Amaranthaceae & A & $3 b$ & Africa $\mathrm{S}$ \\
\hline Amaranthus palmeri S. Watson & Amaranthaceae & A & $3 b$ & N America S, W \\
\hline $\begin{array}{l}\text { Amaranthus powellii S. Watson } \\
\text { [= Amaranthus bouchonii Thell. }]\end{array}$ & Amaranthaceae & A & $3 a$ & N America S; S America N \\
\hline Ambrosia trifida L. & Asteraceae & A & $2 \mathrm{a}$ & N America \\
\hline Ammi majus L. & Apiaceae & A & $2 \mathrm{a}$ & Medit, Asia S, W \\
\hline Amsinckia calycina (Moris) Chater & Boraginaceae & A & $4 \mathrm{a}$ & S America $\mathrm{S}$ \\
\hline Anacyclus radiatus Loisel. & Asteraceae & A & $1 \mathrm{a}$ & Medit W \\
\hline Anthemis austriaca Jacq. & Asteraceae & A & $4 a$ & Asia W; Europe S \\
\hline Artemisia verlotiorum Lamotte & Asteraceae & $\mathrm{P}$ & $?$ & Asia E (China) \\
\hline Asperula arvensis L. & Rubiaceae & A & $2 \mathrm{a}$ & Asia S, W; Africa N \\
\hline Azolla filiculoides Lam. ${ }^{1}$ & Azollaceae & $\mathrm{AP}$ & $4 \mathrm{a}$ & $\begin{array}{l}\mathrm{N} \text { and } \mathrm{S} \text { America (warm and moderate } \\
\text { regions) }\end{array}$ \\
\hline Bidens pilosa $\mathrm{L}$. & Asteraceae & A & $2 \mathrm{a}$ & S America? \\
\hline Bifora radians M. Bieb. $^{2}$ & Apiaceae & A & $4 \mathrm{a}$ & Asia W; Europe S, E \\
\hline $\begin{array}{l}\text { Brachypodium distachyon (L.) P. } \\
\text { Beauv. }\end{array}$ & Poaceae & A & $2 \mathrm{a}$ & Medit \\
\hline Briza minor L. & Poaceae & A & $2 \mathrm{a}$ & Medit \\
\hline Bromus commutatus Schrad. & Poaceae & A & $2 b$ & Europe W, S, E; Africa N; Asia W \\
\hline Bromus grossus Desf. ex DC. & Poaceae & A & $1 \mathrm{a}, 2 \mathrm{a}$ & $\begin{array}{l}\text { Europe (Austria, Belgium, Italy, } \\
\text { Luxembourg, Czech Republic, } \\
\text { Liechtenstein) }\end{array}$ \\
\hline Bromus lanceolatus Roth & Poaceae & A & $2 a$ & Medit \\
\hline Bromus rigidus Roth & Poaceae & A & $2 \mathrm{a}$ & Medit \\
\hline Bromus squarrosus L. & Poaceae & A & $?$ & Europe S; Asia S, W \\
\hline $\begin{array}{l}\text { Bromus briziformis Fisch. et. C. A. } \\
\text { Mey. }\end{array}$ & Poaceae & A & 1a & Asia S, W \\
\hline $\begin{array}{l}\text { Bromus catharticus Vahl } \\
\text { [= Bromus willdenowii Kunth }]\end{array}$ & Poaceae & AP & $4 \mathrm{a}$ & S America \\
\hline Bunium bulbocastanum L. & Apiaceae & $\mathrm{P}$ & $2 \mathrm{a}$ & Europe S, W \\
\hline Bupleurum fontanesii Caruel & Apiaceae & A & $2 \mathrm{a}$ & Medit \\
\hline $\begin{array}{l}\text { Calamintha menthifolia Host } \\
\text { [= Calamintha sylvatica Bromf. }]\end{array}$ & Lamiaceae & $\mathrm{P}$ & $2 \mathrm{a}$ & Medit \\
\hline Camelina rumelica Velen. ${ }^{3}$ & Brassicaceae & A & $3 a$ & Europe E, S; Asia W \\
\hline Carthamus tinctorus L. & Asteraceae & A & $2 a$ & Asia (Middle East, India) \\
\hline Centaurea calcitrapa L. ${ }^{4}$ & Asteraceae & B & $1 \mathrm{a}, 2 \mathrm{a}$ & Medit \\
\hline Centaurea diluta Aiton & Asteraceae & $\mathrm{AP}$ & $2 \mathrm{a}$ & Medit W \\
\hline Centaurea solstitialis L. & Asteraceae & $\mathrm{A}$ & $4 a$ & Medit \\
\hline Centaurea stenolepis A. Kern. & Asteraceae & $\mathrm{P}$ & $4 \mathrm{a}$ & Europe S, E (Anatolia N-W ) \\
\hline
\end{tabular}

Oxbow lakes in the Odra valley in the vicinity of Bytom Odrzański (leg S. Rosadziński 2008, det. K. Latowski, POZ).

At the railway station in Jarocin (Czarna 2005).

On the railway embankment between Drawski Młyn and Krzyż Wielkopolski (Latowski 1981).

The following species were also reported from Wielkopolska: Centaurea decipiens Thuill. and C. pratensis Thuill. (Szulczewski (1951). These species were not included in the checklist due to the lack of comparative materials and subsequent confirmations, as well as some taxonomic doubts. 


\begin{tabular}{|c|c|c|c|c|}
\hline Name of species & Name of family & BF & OP & Native range \\
\hline Chenopodium pratericola Rydb. & Chenopodiaceae & A & $2 \mathrm{a}$ & N America \\
\hline Chenopodium quinoa L. & Chenopodiaceae & $\mathrm{A}$ & $2 \mathrm{a}$ & S America (Ecuador, Peru, Bolivia ) \\
\hline Chenopodium ambrosioides L. & Chenopodiaceae & A & $2 \mathrm{a}$ & S America; N America S \\
\hline Chenopodium berlandieri Moq. & Chenopodiaceae & A & $2 \mathrm{a}$ & N America \\
\hline Chenopodium hircinum Schrader & Chenopodiaceae & $\mathrm{A}$ & $2 \mathrm{a}$ & S America \\
\hline Chenopodium schraderanum Schult. & Chenopodiaceae & A & $?$ & Africa $\mathrm{N}$ \\
\hline Chloris barbata Sw. & Poaceae & $\mathrm{A}$ & $2 \mathrm{a}$ & Asia S, E, tropic \\
\hline Chloris truncata $\mathrm{R} . \mathrm{Br}$. & Poaceae & $\mathrm{B}$ & $2 \mathrm{a}$ & Australia \\
\hline Chloris virgata $\mathrm{Sw}$. & Poaceae & A & $4 a$ & $\begin{array}{l}\text { Eurasia; Africa; and the Americas (the } \\
\text { warmer temperate, subtropical, and } \\
\text { tropical regions) }\end{array}$ \\
\hline Chorispora tenella (Pall.) DC. & Brassicaceae & A & $4 \mathrm{a}$ & Europe S, E; Asia \\
\hline $\begin{array}{l}\text { Citrullus lanatus (Thunb.) Matsum. } \\
\text { et. Nakai }\end{array}$ & Cucurbitaceae & A & $3 a$ & Africa S (Namibia) \\
\hline $\begin{array}{l}\text { Cladanthus mixtus (L.) Oberpr. \& } \\
\text { Vogt } \\
\text { [= Chamaemelum mixtum (L.) All.] }\end{array}$ & Asteraceae & A & $2 \mathrm{a}$ & Medit \\
\hline Claytonia perfoliata Donn ex Willd. & Portulacaceae & A & $4 \mathrm{a}$ & $\mathrm{N}$ America \\
\hline Cochlearia officinalis L. & Brassicaceae & $\mathrm{BP}$ & $2 \mathrm{a}$ & Europe N, W, C \\
\hline $\begin{array}{l}\text { Coincya monensis (L.) Greuter et. } \\
\text { Burdet s. } 1 .\end{array}$ & Brassicaceae & AP & $2 \mathrm{a}$ & Europe S, W \\
\hline Coleostephus myconis (L.) Rchb. f. & Asteraceae & A & $2 \mathrm{a}$ & Medit \\
\hline Commelina coelestis Willd. & Commelianaceae & $\mathrm{P}$ & $2 \mathrm{a}$ & N America $\mathrm{S}$ \\
\hline $\begin{array}{l}\text { Conopodium majus (Gouan) Lorent } \\
\text { Consolida orientalis (J. Gay) }\end{array}$ & Apiaceae & $\mathrm{P}$ & $2 \mathrm{a}$ & Europe W, S \\
\hline $\begin{array}{l}\text { Schrödinger } \\
\text { [= Consolida hispanica (Costa) } \\
\text { Greuter et Burdet }]\end{array}$ & Ranunculaceae & A & $4 a$ & Medit \\
\hline Corispermum nitidum Kit. ex Schult. & Chenopodiaceae & A & $3 a$ & Europe S, E, W; Asia W \\
\hline Coronopus didymus (L.) Sm. & Brassicaceae & A & $2 \mathrm{a}$ & S America \\
\hline Cotula anthemoides L. & Asteraceae & A & $2 \mathrm{a}$ & Africa S; Asia S, W \\
\hline $\begin{array}{l}\text { Crambe abyssinica Hochst. ex R. E. } \\
\text { Fr. }\end{array}$ & Brassicaceae & A & $?$ & Africa S (Abisynia) \\
\hline Crepis setosa Haller $\mathrm{f}$. & Asteraceae & A & $1 \mathrm{a}$ & Europe C, S \\
\hline $\begin{array}{l}\text { Crepis sprengeriana (L.) All. } \\
\text { [= Picris sprengeriana (L.) Poir. }]\end{array}$ & Asteraceae & A & $2 \mathrm{a}$ & Medit \\
\hline Crupina vulgaris Pers. ex Cass. & Asteraceae & A & $1 \mathrm{a}$ & Europe S \\
\hline Cucurbita ficifolia Bouché & Cucurbitaceae & A & $2 \mathrm{a}$ & S America (Andes) \\
\hline Cuscuta australis $\mathrm{R} . \mathrm{Br}$. & Cuscutaceae & A & $3 a$ & Australia; Asia; Europe S \\
\hline Cuscuta campestris Yunck. ${ }^{5}$ & Cuscutaceae & A & $3 a$ & S America \\
\hline $\begin{array}{l}\text { Cuscuta cesatiana Bertol. }{ }^{6} \\
\text { [= Cuscuta arvensis Beyr. var. } \\
\text { calycina Engelm.] }\end{array}$ & Cuscutaceae & A & $3 a$ & Europe S, C to Asia S, W (India) \\
\hline $\begin{array}{l}\text { Cuscuta gronovii Willd. ex Schult. } \\
\text { Cuscuta trifolii Bab, et Gibson }\end{array}$ & Cuscutaceae & A & $1 \mathrm{a}, 2 \mathrm{a}$ & N America \\
\hline $\begin{array}{l}\text { Cuscuta trifolii Bab. et Gibson } \\
\text { [= Cuscuta epithymum (L.) Nathh. } \\
\text { subsp. trifolii (Bab. \& Gibson) Beger] }\end{array}$ & Cuscutaceae & A & $?$ & Europe W, S \\
\hline Cynosurus echinatus L. & Poaceae & A & $2 \mathrm{a}$ & Medit \\
\hline Cyperus congestus Vahl & Cyperaceae & $\mathrm{P}$ & $2 \mathrm{a}$ & Africa $S$ \\
\hline $\begin{array}{l}\text { Cytisus elongatus Waldst. \& Kit. } \\
\text { [= Chamaecytisus glaber (L.f.) } \\
\text { Rothm.] }\end{array}$ & Fabaceae & $\mathrm{T}$ & $2 \mathrm{a}$ & $\begin{array}{l}\text { Europe S (Serbia, Montenegro, Bulgaria, } \\
\text { Romania) }\end{array}$ \\
\hline
\end{tabular}




\begin{tabular}{|c|c|c|c|c|}
\hline Name of species & Name of family & BF & $\mathbf{O P}$ & Native range \\
\hline $\begin{array}{l}\text { Dactyloctenium aegyptium (L.) } \\
\text { Willd. }\end{array}$ & Poaceae & A & $2 \mathrm{a}$ & Africa tropic, Asia $\mathrm{S}$ \\
\hline Dasypyrum villosum (L.) Borbás & Poaceae & A & $2 \mathrm{a}$ & Europe E, S; Asia W \\
\hline $\begin{array}{l}\text { Dimorphotheca pluvialis (L.) } \\
\text { Moench }\end{array}$ & Asteraceae & AP & $2 \mathrm{a}$ & Africa $S$ \\
\hline Draba muralis L. & Brassicaceae & A & $2 \mathrm{a}$ & Europe; Africa N, W; Asia W \\
\hline Dracocephalum moldavica L. & Lamiaceae & A & $2 \mathrm{a}$ & $\begin{array}{l}\text { Asia (China, Sibirien, Central Asia), } \\
\text { Europe E }\end{array}$ \\
\hline Dracocephalum thymiflorum L. & Lamiaceae & A & $3 a$ & Asia W, C; Europe E \\
\hline Echium plantagineum L. & Boraginaceae & $\mathrm{AB}$ & $2 \mathrm{a}$ & Europe S, W; Africa N; Asia S, E \\
\hline Elymus canadensis L. & Poaceae & $\mathrm{P}$ & $4 \mathrm{a}$ & N America \\
\hline Eragrostis mexicana (Hornem.) Link & Poaceae & A & $2 \mathrm{a}$ & S America; N America \\
\hline Eragrostis multicaulis Steud. & Poaceae & A & $2 \mathrm{a}$ & Asia E; Europe S; Africa N \\
\hline $\begin{array}{l}\text { Eragrostis cilianensis (All.) Vignolo } \\
\text { ex Janch. }\end{array}$ & Poaceae & A & $4 \mathrm{a}$ & Eurasia; Africa \\
\hline $\begin{array}{l}\text { Erucastrum nasturtiifolium (Poir.) } \\
\text { O. E. Schulz }\end{array}$ & Brassicaceae & $\mathrm{BP}$ & $3 a$ & Europe S, W \\
\hline Erysimum crepidifolium Rchb. & Brassicaceae & $\mathrm{BP}$ & $2 \mathrm{a}$ & Europe C, S \\
\hline Erysimum diffusum Ehrh. & Brassicaceae & $\mathrm{B}$ & $?$ & Europe S, E, C; Asia W \\
\hline Euphorbia platyphyllos L. & Euphorbiaceae & A & $2 \mathrm{a}$ & Europe (medit and subatl) \\
\hline Fumaria capreolata L. & Fumariaceae & A & $2 \mathrm{a}$ & Medit \\
\hline Fumaria densiflora DC. & Fumariaceae & A & $2 \mathrm{a}$ & Europe S (Medit); Asia C, W \\
\hline Galeopsis segetum Neck. & Lamiaceae & A & $2 \mathrm{a}, 4 \mathrm{a}$ & Europe S (Medit) \\
\hline Galium parisiense L. & Rubiaceae & A & $2 \mathrm{a}$ & Europe S, W, Africa N, Asia Minor \\
\hline Galium tricornutum Dandy ${ }^{7}$ & Rubiaceae & A & $1 \mathrm{a}, 2 \mathrm{a}, 3 \mathrm{a}$ & Europe N, W; Africa N; Asia W \\
\hline Gaudinia fragilis (L.) P. Beauv. & Poaceae & A & $2 \mathrm{a}$ & Medit \\
\hline Geranium rotundifolium L. & Geraniaceae & A & $3 a ?$ & Europe S, W; Asia S; Africa N \\
\hline Glaucium flavum Crantz & Papaveraceae & A & $2 \mathrm{a}$ & Medit \\
\hline Glaucium corniculatum (L.) Rudolph & Papaveraceae & A & $2 \mathrm{a}, 3 \mathrm{a}$ & Medit \\
\hline Guizotia abyssinica (L. f.) Cass. & Asteraceae & A & $2 \mathrm{a}$ & Africa E (Ethiopia) \\
\hline Gypsophila perfoliata L. & Caryophyllaceae & $\mathrm{P}$ & $4 \mathrm{a}$ & Europe E; Asia W \\
\hline Heliotropium europaeum L. & Boraginaceae & A & $2 \mathrm{a}, 4 \mathrm{a}$ & Medit \\
\hline Herniaria incana Lam. & Caryophyllaceae & $\mathrm{P}$ & $4 \mathrm{~b}$ & Europe (Medit) to Asia W \\
\hline $\begin{array}{l}\text { Hirschfeldia incana (L.) Lagr.-Foss. } \\
\text { [= Hirschfeldia adpressa Moench }]\end{array}$ & Brassicaceae & A & $4 \mathrm{a}$ & Medit \\
\hline Hordeum secalinum Schreb. & Poaceae & $\mathrm{P}$ & $2 \mathrm{a}$ & Europe W, S to Asia Minor; Africa N \\
\hline Humulus scandens (Lour.) Merr. & Cannabaceae & A & $2 \mathrm{a}$ & Asia E \\
\hline $\begin{array}{l}\text { Hygrophila polysperma (Roxb.) T. } \\
\text { Anderson }^{8}\end{array}$ & Acanthaceae & $\mathrm{P}$ & $4 \mathrm{a}$ & Asia S, E (India, Malaysia) \\
\hline Hypericum mutilum L. & Hypericaceae & A & 1a & N America \\
\hline $\begin{array}{l}\text { Hypericum gymnanthum Engelm. \& } \\
\text { A. Gray }\end{array}$ & Hypericaceae & A & $1 \mathrm{a}$ & N America \\
\hline $\begin{array}{l}\text { Juncus anthelatus (Wiegand) R. E. } \\
\text { Brooks \& Whittem. }\end{array}$ & Juncaceae & $\mathrm{P}$ & $4 a$ & N America E; C America (Mexico) \\
\hline Lactuca tatarica (L.) C. A. Mey & Asteraceae & $\mathrm{P}$ & $?$ & Europe E; Asia E to N. \& W. America N \\
\hline Lactuca virosa $L$. & Asteraceae & $\mathrm{AB}$ & $1 \mathrm{a}, 3 \mathrm{~b}$ & Medit \\
\hline Lathyrus aphaca L. & Fabaceae & A & $4 \mathrm{a}$ & Europe W, C, S; Asia W; Africa N \\
\hline $\begin{array}{l}\text { Legousia speculum-veneris (L.) } \\
\text { Chaix }\end{array}$ & Campanulaceae & A & $2 \mathrm{a}$ & Medit \\
\hline Lepidium latifolium L. & Brassicaceae & $\mathrm{P}$ & $4 b$ & Medit, Asia S, W \\
\hline Lepidium perfoliatum L. & Brassicaceae & A & $4 a$ & Europe E; Asia \\
\hline Linaria repens (L.) Mill. & Scrophulariaceae & $\mathrm{P}$ & $4 \mathrm{a}$ & Europe (Medit-Atl) \\
\hline
\end{tabular}

\footnotetext{
7 In the highland belt and in the Lower Silesia, this species occurs as an archaeophyte Zając 1979; Zając et al. 2009.). In Wielkopolska, it appeared only ephemerally in Poznan (Żukowski 1959).
} 


\begin{tabular}{|c|c|c|c|c|}
\hline Name of species & Name of family & $\mathbf{B F}$ & OP & Native range \\
\hline Linum austriacum L. & Linaceae & $\mathrm{P}$ & $?$ & $\begin{array}{l}\text { Europe E, S, C; Africa N; Asia W } \\
\text { (Turkey) }\end{array}$ \\
\hline Lolium rigidum Gaudin & Poaceae & A & $2 \mathrm{a}$ & Medit, Asia S, W \\
\hline Lythrum junceum Banks \& Sol. & Lythraceae & $\mathrm{P}$ & $2 \mathrm{a}$ & Medit \\
\hline Malva nicaeensis All. ${ }^{9}$ & Malvaceae & A & $2 \mathrm{a}$ & Medit \\
\hline Marrubium peregrinum L. & Lamiaceae & $\mathrm{P}$ & $2 \mathrm{a}$ & Europe S, E; Asia Minor \\
\hline Medicago arabica (L.) Huds. & Fabaceae & A & $1 \mathrm{a}$ & Medit \\
\hline Medicago polymorpha L. & Fabaceae & A & $?$ & Medit \\
\hline Melica picta K. Koch ${ }^{10}$ & Poaceae & $\mathrm{P}$ & $3 a$ & Europe C, E \\
\hline Melilotus indicus (L.) All. & Fabaceae & A & $2 \mathrm{a}$ & Medit, Asia S, W \\
\hline $\begin{array}{l}\text { Mimulus moschatus Douglas ex } \\
\text { Lindl. }\end{array}$ & Scrophulariaceae & $\mathrm{P}$ & $?$ & N America W \\
\hline Minuartia hybrida (Vill.) Schischk. & Caryophyllaceae & A & $1 \mathrm{a}$ & Medit, Asia S, W \\
\hline Moenchia mantica (L.) Bartl. & Caryophyllaceae & A & $2 \mathrm{a}$ & Europe S, E \\
\hline Myagrum perfoliatum L. & Brassicaceae & A & $4 a$ & Europe S; Asia W \\
\hline Nicotiana rustica L. & Solanaceae & A & $2 \mathrm{a}$ & America $\mathrm{C}, \mathrm{S}$ \\
\hline Nicotiana tabacum L. & Solanaceae & A & $2 \mathrm{a}$ & America $\mathrm{C}, \mathrm{S}$ ? \\
\hline Oenothera $\times$ tacikii Rostański & Onagraceae & $\mathrm{B}$ & $4 a$ & Europe \\
\hline Oenothera cruciata Nutt. ex Don & Onagraceae & $\mathrm{B}$ & $4 \mathrm{a}$ & $\mathrm{N}$ America \\
\hline $\begin{array}{l}\text { Oenothera fallax Renner em. } \\
\text { Rostański }\end{array}$ & Onagraceae & $\mathrm{B}$ & $4 a$ & America N \\
\hline Oenothera flaemingina Hudziok & Onagraceae & $\mathrm{B}$ & $4 a$ & Europe \\
\hline Oenothera parviflora L. & Onagraceae & $\mathrm{B}$ & $4 \mathrm{a}$ & $\mathrm{N}$ America \\
\hline $\begin{array}{l}\text { Oenothera pycnocarpa G. F. Atk. et } \\
\text { Bartlett }\end{array}$ & Onagraceae & B & $4 a$ & N America \\
\hline Orobanche ramosa L. & Orobanchaceae & A & $2 \mathrm{a}, 3 \mathrm{a}$ & Africa, Asia W, Europe \\
\hline $\begin{array}{l}\text { Oxalis tetraphylla Cav. } \\
\text { [= Oxalis deppei Lodd. ex Sweet }]\end{array}$ & Oxalidaceae & $\mathrm{P}$ & $4 b$ & America C (Mexico) \\
\hline Panicum capillare L. & Poaceae & A & $4 b$ & N America \\
\hline $\begin{array}{l}\text { Panicum dichotomiflorum Michx. } \\
\text { Papaver albiflorum (Boiss.) }\end{array}$ & Poaceae & A & $?$ & S America; N America \\
\hline $\begin{array}{l}\text { Paczoski }^{11} \\
\text { [= Papaver dubium L. subsp. } \\
\text { albiflorum] }\end{array}$ & Papaveraceae & A & $4 a$ & Europe S, W (medit) \\
\hline Paspalum racemosum Lam. & Poaceae & A & $2 \mathrm{a}$ & S America W \\
\hline Petrorhagia saxifraga (L.) Link & Caryophyllaceae & $P$ & $2 \mathrm{a}$ & Europe S, C; Asia W \\
\hline Phalaris canariensis L. & Poaceae & A & $4 a$ & Medit \\
\hline Phalaris coerulescens Desf. & Poaceae & $\mathrm{P}$ & $2 \mathrm{a}$ & Medit \\
\hline Plantago afra $L .{ }^{12}$ & Plantaginaceae & A & $4 \mathrm{a}$ & Africa C, N; Asia S, W; Europe S, E \\
\hline Plantago lagopus L. & Plantaginaceae & A & $2 \mathrm{a}$ & Medit \\
\hline Polycarpon tetraphyllum (L.) L. & Caryophyllaceae & A & $2 \mathrm{a}$ & Europe (medit) \\
\hline Polygonum patulum M. Bieb. & Polygonaceae & A & $2 \mathrm{a}$ & $\begin{array}{l}\text { Europe W, Medit, Asia C (Caucas, } \\
\text { Siberia, Mongolia, China) }\end{array}$ \\
\hline Polypogon monspeliensis (L.) Desf. & Poaceae & A & $2 \mathrm{a}$ & Medit, Asia S, W \\
\hline Potentilla intermedia L. & Rosaceae & $\mathrm{BP}$ & $2 \mathrm{a}$ & Europe N, W; Africa N; Asia (Siberia) \\
\hline Rapistrum perenne (L.) All. & Brassicaceae & $\mathrm{P}$ & $1 \mathrm{a}, 3 \mathrm{a}$ & Europe E, S; Asia W \\
\hline Rapistrum rugosum (L.) All. & Brassicaceae & A & $1 \mathrm{a}, 2 \mathrm{a}, 3 \mathrm{a}$ & Medit \\
\hline Reseda alba $\mathrm{L}$. & Resedaceae & AP & $1 \mathrm{a}, 2 \mathrm{a}$ & Medit \\
\hline Ricinus communis L. & Euphorbiaceae & A & $3 a ?$ & Africa tropic, Middle East \\
\hline
\end{tabular}

Reported for the first time from the vicinity of Konin (Gąbka \& Owsianny 2009).

Malva mauritiana L. - reported from Wielkopolska (Szulczewski 1951; Czarna 2009, Celka 2014 POZ), in this work it was included in M. sylvestris L. Native to Poland, occurs only in a single locality in the south-eastern part of the country (Zając \& Zając 2001, Oklejewicz et al. 2015).

Railway tracks at the railway station in Kościan (Czarna 2009).

Observed in Kórnik near Poznań in the years 2014-2015; in total, a few individuals occurred (leg. A. Wysocki, det. K. Latowski; POZ); in 2016 - not found. 


\begin{tabular}{|c|c|c|c|c|}
\hline Name of species & Name of family & BF & OP & Native range \\
\hline Roemeria hybrida (L.) DC. & Papaveraceae & A & $4 a$ & Medit, Asia W \\
\hline Rosa gorenkensis Besser & Rosaceae & $\mathrm{T}$ & $4 a$ & Europe S, W; Asia W, \\
\hline Rumex bucephalophorus L. & Polygonaceae & A & $2 \mathrm{a}$ & Medit \\
\hline Rumex longifolius DC. & Polygonaceae & $P$ & $?$ & Asia W, C \\
\hline $\begin{array}{l}\text { Rumex salicifolius Weinm. } \\
\text { [= Rumex triangulivalvis (Danser) } \\
\text { Rech. f.] }\end{array}$ & Polygonaceae & $\mathrm{P}$ & $3 a$ & N America \\
\hline Rumex stenophyllus Ledeb. ${ }^{13}$ & Polygonaceae & $\mathrm{P}$ & $4 a$ & Europe S, W, Azja C (Siberia) \\
\hline Salsola collina Pall. & Chenopodiaceae & A & $3 a$ & $\begin{array}{l}\text { Asia (Korea, Mongolia, China, Pakistan, } \\
\text { Russia, Central Asia); Europe E }\end{array}$ \\
\hline Salsola soda L. & Chenopodiaceae & A & $3 a$ & Medit \\
\hline $\begin{array}{l}\text { Salvia dumetorum Andrz. ex Besser } \\
\text { [= Salvia dumetorum Andrz.] }\end{array}$ & Lamiaceae & $\mathrm{P}$ & $2 \mathrm{a}$ & Europe S, E; Asia C \\
\hline Scirpus georgianus R. M. Harper & Cyperaceae & $\mathrm{P}$ & $4 a$ & N America \\
\hline Senecio inaequidens DC. & Asteraceae & $\mathrm{P}$ & $4 a$ & $\begin{array}{l}\text { Africa (South Africa, Lesotho, } \\
\text { Swaziland) }\end{array}$ \\
\hline $\begin{array}{l}\text { Sesamoides interrupta (Boreau) G. } \\
\text { López } \\
\text { [=Sesamoides canescens (L.) Kuntze] }\end{array}$ & Resedaceae & $\mathrm{P}$ & $3 a$ & Medit W \\
\hline Sideritis montana L. & Lamiaceae & A & $4 a$ & Medit, Asia W \\
\hline $\begin{array}{l}\text { Silene gallinyi Heuff. ex Rchb. } \\
\text { [= Silene trinervia Sebast. \& Mauri] }\end{array}$ & Caryophyllaceae & $\mathrm{P}$ & $2 \mathrm{a}$ & Europe (Balkans) \\
\hline Silene linicola C. C. Gmel. & Caryophyllaceae & A & $2 \mathrm{a}$ & Europe W (France) \\
\hline Silene scabriflora Brot. & Caryophyllaceae & A & $2 \mathrm{a}$ & Europe S, W \\
\hline Sisymbrium austriacum Jacq. & Brassicaceae & $\mathrm{BP}$ & $2 \mathrm{a}$ & Europe $\mathrm{C}, \mathrm{S}, \mathrm{W}$ \\
\hline Sisymbrium orientale L. & Brassicaceae & $\mathrm{ABP}$ & $2 \mathrm{a}, 3 \mathrm{a}$ & Europe S; Asia W \\
\hline Solanum alatum Moench & Solanaceae & A & $3 a$ & Medit, Asia Minor \\
\hline $\begin{array}{l}\text { Solanum angustifolium Mill. } \\
\text { [= Solanum cornutum Lam.] }\end{array}$ & Solanaceae & A & $3 a$ & N America S \\
\hline Solanum triflorum Nutt. & Solanaceae & A & $2 \mathrm{a}$ & S America; N America \\
\hline Sorghum bicolor (L.) Moench & Poaceae & A & $3 a$ & Africa tropic \\
\hline Sorghum halepense (L.) Pers. & Poaceae & $P$ & $3 a$ & Europe (Medit) to Asia C \\
\hline $\begin{array}{l}\text { Thlaspi alliaceum L. } \\
\text { Thlaspi caerulescens J. Presl et. C. }\end{array}$ & Brassicaceae & A & $?$ & Africa N, E; Asia W; Europe S, E \\
\hline $\begin{array}{l}\text { Presl } \\
{[=\text { Thlaspi alpestre L. }]}\end{array}$ & Brassicaceae & $\mathrm{BP}$ & $2 a$ & Europe W, C \\
\hline Thlaspi perfoliatum $\mathrm{L}$. & Brassicaceae & A & $4 a$ & Europe S; Asia W \\
\hline Urtica cannabina L. & Urticaceae & $\mathrm{P}$ & $3 a$ & Europe E (Russia), Asia W, C (to China) \\
\hline Valerianella carinata Loisel. & Valerianaceae & $\mathrm{A}$ & $1 \mathrm{a}$ & Medit? \\
\hline Veronica peregrina $\mathrm{L}$. & Scrophulariaceae & $\mathrm{AB}$ & $4 a$ & N America S \\
\hline Vicia articulata Hornem. & Fabaceae & $\mathrm{A}$ & $2 \mathrm{a}$ & Medit \\
\hline Vicia bithynica (L.) L. & Fabaceae & A & $2 a$ & Medit \\
\hline Vicia lutea L. & Fabaceae & A & $3 a$ & Europe; Asia W; Africa N \\
\hline Vicia pannonica Crantz & Fabaceae & A & $4 a$ & Europe S, C; Asia W \\
\hline Viola ambigua Waldst. \& Kit. & Violaceae & $\mathrm{P}$ & $3 a ?$ & Europe C, S, E (Caucasus) \\
\hline Vulpia geniculata (L.) Link & Poaceae & A & $2 \mathrm{a}$ & Europe $\mathrm{S}$; Africa N \\
\hline $\begin{array}{l}\text { Xanthium orientale L. } \\
\text { [= Xanthium macrocarpum DC. }]\end{array}$ & Asteraceae & A & $2 \mathrm{a}$ & N America? \\
\hline Xanthium spinosum L. & Asteraceae & A & $2 \mathrm{a}$ & S America \\
\hline
\end{tabular}

Explanations: BF - biological form, A - annual plants, B - biennial plants, $\mathrm{P}$ - perennial, herbaceous plants, $\mathrm{S}$ - dwarf shrubs and sub-shrubs, $\mathrm{T}$ - shrubs and trees; OP - observation period, 1 - to 1901,2 - 1901-1951,3 - 1951-2001, 4 - after 2001, a - species was observed once, b-species was observed repeatedly; Native range: $\mathrm{N}$ - north, $\mathrm{W}$ - west, $\mathrm{E}$ - east, $\mathrm{S}$ - south, $\mathrm{C}$ - central, Medit - Mediterranean, Atl - Atlantic; ? - no data information uncertain 
Appendix 2. Checklist of casual alien species of Wielkopolska - ergasiophygophytes

\begin{tabular}{|c|c|c|c|}
\hline Name of species & Name of family & BF & OP \\
\hline $\begin{array}{l}\text { Abies concolor (Gord. et Glend.) Lindl. ex } \\
\text { Hildebr. }\end{array}$ & Pinaceae & $\mathrm{T}$ & $4 \mathrm{~b}$ \\
\hline Acer saccharinum L. & Aceraceae & $\mathrm{T}$ & $4 \mathrm{~b}$ \\
\hline Acer tataricum L. ${ }^{1}$ & Aceraceae & $\mathrm{T}$ & $4 b$ \\
\hline Achillea tomentosa L. & Asteraceae & $\mathrm{P}$ & $?$ \\
\hline Adonis annua L. em. Huds. & Ranunculaceae & A & $2 \mathrm{a}$ \\
\hline Aesculus $\times$ carnea Hayne & Hippocastanaceae & $\mathrm{T}$ & $4 b$ \\
\hline Aesculus flava Sol. & Hippocastanaceae & $\mathrm{T}$ & $3 a$ \\
\hline Alcea rosea L. & Malvaceae & $\mathrm{BP}$ & $4 \mathrm{~b}$ \\
\hline Allium carinatum L. & Liliaceae & $\mathrm{P}$ & $2 \mathrm{a}$ \\
\hline Allium cepa L. & Liliaceae & $\mathrm{P}$ & $4 a$ \\
\hline Allium fistulosum L. & Liliaceae & $\mathrm{P}$ & $2 \mathrm{a}$ \\
\hline Allium giganteum Regel & Liliaceae & $\mathrm{P}$ & $4 a$ \\
\hline Allium moly L. & Liliaceae & $\mathrm{P}$ & $4 a$ \\
\hline Allium sativum $\mathrm{L}$. & Liliaceae & $\mathrm{P}$ & $2 \mathrm{a}$ \\
\hline Allium schoenoprasum L. & Liliaceae & $\mathrm{P}$ & $4 a$ \\
\hline Allium sphaerocephalon L. & Liliaceae & $\mathrm{P}$ & $2 \mathrm{a}$ \\
\hline $\begin{array}{l}\text { Allium ampeloprasum L. s. } 1 . \\
\text { [= Allium porrum L.] }\end{array}$ & Liliaceae & $\mathrm{P}$ & $2 \mathrm{a}$ \\
\hline Althaea officinalis L. ${ }^{2}$ & Malvaceae & $\mathrm{P}$ & $4 a$ \\
\hline Alyssum saxatile L. ${ }^{3}$ & Brassicaceae & $\mathrm{S}$ & $4 a$ \\
\hline Amaranthus caudatus L. & Amaranthaceae & A & $4 \mathrm{a}$ \\
\hline $\begin{array}{l}\text { Amaranthus cruentus L. } \\
\text { [= Amaranthus paniculatus L.] }\end{array}$ & Amaranthaceae & $\mathrm{A}$ & $4 \mathrm{a}$ \\
\hline Amelanchier lamarckii F. G. Schroed. & Rosaceae & $\mathrm{T}$ & $4 a$ \\
\hline Ammophila arenaria (L.) Link ${ }^{4}$ & Poaceae & $\mathrm{P}$ & $4 a$ \\
\hline Amorpha fruticosa L. & Fabaceae & $\mathrm{T}$ & $4 a$ \\
\hline Anethum graveolens L. & Apiaceae & A & $4 a$ \\
\hline Antirrhinum majus L. & Scrophulariaceae & AP & $4 a$ \\
\hline Apium graveolens $\mathrm{L}$. & Apiaceae & $\mathrm{A}$ & $4 \mathrm{a}$ \\
\hline $\begin{array}{l}\text { Aquilegia caerulea E. James } \\
{[=\text { Aquilegia } \times \text { hybrida hort. }]}\end{array}$ & Ranunculaceae & $\mathrm{P}$ & $4 \mathrm{a}$ \\
\hline Arabis alpina $L .{ }^{5}$ & Brassicaceae & $\mathrm{P}$ & $4 a$ \\
\hline Arabis caucasica Willd. & Brassicaceae & $\mathrm{P}$ & $4 a$ \\
\hline Aristolochia macrophylla Lam. & Aristolochiaceae & $\mathrm{T}$ & $4 a$ \\
\hline Artemisia abrotanum L. & Asteraceae & S & $2 b$ \\
\hline Artemisia dracunculus L. & Asteraceae & $\mathrm{P}$ & $4 b$ \\
\hline $\begin{array}{l}\text { Artemisia ludoviciana Nutt. }{ }^{6} \\
\text { [= Artemisia platyphylla Rydb.] }\end{array}$ & Asteraceae & $\mathrm{P}$ & $3 a$ \\
\hline Artemisia maritima L. & Asteraceae & $\mathrm{S}$ & 1a \\
\hline Artemisia pontica $\mathrm{L} .{ }^{7}$ & Asteraceae & $\mathrm{P}$ & $1 \mathrm{a}$ \\
\hline Aster $\times$ salignus Willd. & Asteraceae & $\mathrm{P}$ & $4 a$ \\
\hline Avena sativa L. & Poaceae & $\mathrm{A}$ & $4 b$ \\
\hline
\end{tabular}

Some information about Acer tataricum L. was attributed to A. ginnala Maxim. [Acer tataricum L. subsp. ginnala (Maxim.) Wesm.]. Probably a synanthropic species. There are some doubts on its status on saline soils (see Zając \& Zając 2001; Mirek et al. 2002).

In Poland, it is a native species in the Pieniny Mountains (Zając \& Zając 2001). In lowlands, this species is cultivated and running wild from cultivation. 4 In Poland, it is a native species only on the Baltic Sea (Zając \& Zając 2001). In addition, this species is cultivated in lowlands and running wild from cultivation.

5 In Poland, it is a native species in the Tatras and on the Babia Góra Mt. (Zając \& Zając 2001). In addition, this species is cultivated in lowlands and runing wild from cultivation.

The species was reported for the first time for Poland from the area of the Wielkopolski National Park. The specimens collected there belong to two varieties: var. ludoviciana and var. incompta (Nutt.) Cronq. (Żukowski et al. 1995).

7 In Poland, it is a native species only in the south-eastern part of Nidziańska Basin (Kaźmierczakowa 2014a). In other parts of Poland, only anthropogenic populations occur (Żukowski \& Piaszyk 1971; Zając \& Zając 2001; Kaźmierczakowa 2014a). In Wielkopolska, it was collected by Spribille (1892, POZ) on the Lake Tuczno near Inowrocław (Żukowski \& Piaszyk 1971). 


\begin{tabular}{|c|c|c|c|}
\hline Name of species & Name of family & $\mathbf{B F}$ & OP \\
\hline Berberis thunbergii DC. ${ }^{8}$ & Berberidaceae & $\mathrm{T}$ & $4 \mathrm{~b}$ \\
\hline Bergenia crassifolia (L.) Fritsch & Saxifragaceae & $\mathrm{P}$ & $4 a$ \\
\hline Beta vulgaris $\mathrm{L}$. & Chenopodiaceae & AP & $4 \mathrm{a}$ \\
\hline Betula lenta L. ${ }^{9}$ & Betulaceae & $\mathrm{T}$ & $4 \mathrm{a}$ \\
\hline Bidens ferulifolia (Jacq.) DC. & Asteraceae & $\mathrm{AB}$ & $4 \mathrm{a}$ \\
\hline Borago officinalis L. & Boraginaceae & A & $4 \mathrm{a}$ \\
\hline Brassica napus L. subsp. napus & Brassicaceae & $\mathrm{AB}$ & $4 \mathrm{~b}$ \\
\hline Buddleja davidii Franch. & Buddlejaceae & $\mathrm{T}$ & $4 \mathrm{a}$ \\
\hline Calandrinia pilosiuscula DC. & Portulacaceae & $\mathrm{AP}$ & $1 \mathrm{a}$ \\
\hline Calendula arvensis $\mathrm{L}$. & Asteraceae & A & $?$ \\
\hline Calendula officinalis L. & Asteraceae & $\mathrm{AP}$ & $4 a$ \\
\hline Callistephus chinensis (L.) Nees & Asteraceae & A & $?$ \\
\hline Campanula carpatica Jacq. & Campanulaceae & $\mathrm{P}$ & $4 a$ \\
\hline Campanula rapunculus L. & Campanulaceae & $\mathrm{B}$ & $2 b$ \\
\hline Cannabis sativa $\mathrm{L}$. & Cannabaceae & A & $4 a$ \\
\hline Capsicum annuum L. & Solanaceae & A & $2 \mathrm{a}$ \\
\hline Caragana arborescens Lam. & Fabaceae & $\mathrm{T}$ & $4 \mathrm{~b}$ \\
\hline Carthamus lanatus L. & Asteraceae & A & $2 \mathrm{a}$ \\
\hline Carya ovata (Mill.) K. Koch & Juglandaceae & $\mathrm{T}$ & $4 a$ \\
\hline Celastrus orbiculatus Thunb. ${ }^{10}$ & Celastraceae & $\mathrm{P}$ & $4 a$ \\
\hline Centaurea dealbata Willd. & Asteraceae & $\mathrm{P}$ & $4 a$ \\
\hline Centaurea montana L. & Asteraceae & $\mathrm{P}$ & $2 \mathrm{a}$ \\
\hline Cerastium tomentosum L. & Caryophyllaceae & $\mathrm{P}$ & $4 \mathrm{~b}$ \\
\hline $\begin{array}{l}\text { Chaenomeles japonica (Thunb.) Lindl. ex } \\
\text { Spach }\end{array}$ & Rosaceae & $\mathrm{T}$ & $4 a$ \\
\hline $\begin{array}{l}\text { Chamaemelum nobile (L.) All. } \\
\text { [= Anthemis nobilis L.] }\end{array}$ & Asteraceae & $\mathrm{P}$ & $2 \mathrm{a}$ \\
\hline Chenopodium capitatum (L.) Asch. & Chenopodiaceae & A & $2 \mathrm{a}$ \\
\hline Cicerbita macrophylla (Willd.) Wallr. ${ }^{11}$ & Asteraceae & $\mathrm{P}$ & $4 a$ \\
\hline Clarkia amoena (Lehm.) A. Nelson \& J. F. & & & \\
\hline $\begin{array}{l}\text { Macbr. subsp. lindleyi (Douglas) H. F. Lewis \& } \\
\text { M. R. Lewis } \\
\text { [= Godetia grandiflora Lindl.] }\end{array}$ & Onagraceae & A & $4 a ?$ \\
\hline Collomia grandiflora Douglas & Polemoniaceae & A & $2 \mathrm{a}$ \\
\hline Colutea arborescens L. & Fabaceae & $\mathrm{T}$ & $4 a$ \\
\hline Colutea media Willd. ${ }^{12}$ & Fabaceae & $\mathrm{T}$ & $4 a$ \\
\hline Consolida ajacis (L.) Schur & Ranunculaceae & A & $4 \mathrm{a}$ \\
\hline Convolvulus tricolor L. & Convolvulaceae & A & $2 \mathrm{a}$ \\
\hline Coriandrum sativum L. & Apiaceae & A & $4 \mathrm{a}$ \\
\hline Cornus mas L. & Cornaceae & $\mathrm{T}$ & $4 \mathrm{a}$ \\
\hline Cosmos bipinnatus Cav. & Asteraceae & A & $4 b$ \\
\hline $\begin{array}{l}\text { Cotoneaster divaricatus Rehder et E. H. } \\
\text { Wilson }\end{array}$ & Rosaceae & $\mathrm{T}$ & $4 \mathrm{a}$ \\
\hline Cotoneaster integerrimus Medik. & Rosaceae & $\mathrm{T}$ & $?$ \\
\hline $\begin{array}{l}\text { Cotoneaster villosulus (Rehder \& E. H. } \\
\text { Wilson) Flinck \& B. Hylm }\end{array}$ & Rosaceae & $\mathrm{T}$ & $4 \mathrm{a}$ \\
\hline Crataegus pedicellata Sarg. & Rosaceae & $\mathrm{T}$ & $4 \mathrm{a}$ \\
\hline Crataegus coccinea $\mathrm{L}$. & Rosaceae & $\mathrm{T}$ & $4 a$ \\
\hline Cucumis sativus L. & Cucurbitaceae & A & $4 b$ \\
\hline
\end{tabular}

Reported from the Wielkopolski National Park (Purcel 2009).

Planted and running wild in the acidic oak forest in the Gołąbki Forest District (leg. and det. Chmiel 1987, POZ).

Wielkopolski National Park (Danielewicz \& Maliński 1995; Purcel 2009).

Found in the village Dobrzyca (Czarna 2003, 2007).

Wielkopolski National Park (Purcel 2009). 


\begin{tabular}{|c|c|c|c|}
\hline Name of species & Name of family & BF & OP \\
\hline Cucurbita moschata Duchesne & Cucurbitaceae & A & $4 \mathrm{a}$ \\
\hline Cucurbita pepo L. & Cucurbitaceae & A & $4 b$ \\
\hline Dianthus barbatus L. & Caryophyllaceae & $\mathrm{P}$ & $4 b$ \\
\hline Dicentra spectabilis L. & Fumariaceae & $\mathrm{P}$ & $4 b$ \\
\hline Dipsacus sativus (L.) Honck. & Dipsacaceae & $\mathrm{BP}$ & $4 a$ \\
\hline Doronicum columnae Ten. & Asteraceae & $\mathrm{P}$ & $4 \mathrm{a}$ \\
\hline Doronicum pardalianches L. & Asteraceae & $\mathrm{P}$ & $4 a$ \\
\hline Echinacea purpurea (L.) Moench & Asteraceae & $\mathrm{P}$ & $4 a$ \\
\hline Echinops exaltatus Schrad. & Asteraceae & $\mathrm{P}$ & $4 \mathrm{a}$ \\
\hline Elaeagnus commutata Bernh. ex Rydb. & Eleagnaceae & $\mathrm{T}$ & $4 a$ \\
\hline $\begin{array}{l}\text { Eruca sativa Mill. } \\
\text { [= Eruca vesicaria (L.) Cav. subsp. sativa } \\
\text { (Mill.) Thell.] }\end{array}$ & Brassicaceae & A & $2 \mathrm{a}$ \\
\hline $\begin{array}{l}\text { Erysimum cheiri (L.) Crantz } \\
{[=\text { Cheiranthus } \times \text { cheiri L. }]}\end{array}$ & Brassicaceae & $\mathrm{S}$ & $2 a$ \\
\hline Eschscholzia californica Cham. & Papaveraceae & AP & $4 \mathrm{a}$ \\
\hline Euphorbia lathyris L. & Euphorbiaceae & $\mathrm{AB}$ & $4 a$ \\
\hline Euphorbia marginata Pursh & Euphorbiaceae & A & $4 b$ \\
\hline Fagopyrum esculentum Moench & Polygonaceae & A & $4 \mathrm{~b}$ \\
\hline Fagopyrum tataricum (L.) Gaertn. & Polygonaceae & A & $4 a$ \\
\hline $\begin{array}{l}\text { Fallopia baldschuanica (Regel) Holub } \\
\text { [= Fallopia aubertii (L. Henry) Holub }]\end{array}$ & Polygonaceae & $\mathrm{P}$ & $4 \mathrm{a}$ \\
\hline Foeniculum vulgare Mill. & Apiaceae & $\mathrm{P}$ & $4 a$ \\
\hline Fragaria $\times$ ananassa Duchesne ex Rozier & Rosaceae & $\mathrm{P}$ & $4 b$ \\
\hline Fraxinus angustifolia Vahl. & Oleaceae & $\mathrm{T}$ & $4 a$ \\
\hline Gaillardia pulchella Foug. & Asteraceae & A & $4 a$ \\
\hline Galium anisophyllon Vill. ${ }^{13}$ & Rubiaceae & $\mathrm{B}$ & $2 \mathrm{a}$ \\
\hline Gypsophila repens L. ${ }^{14}$ & Caryophyllaceae & $\mathrm{P}$ & $4 a$ \\
\hline Helianthus annuus L. & Asteraceae & A & $4 a$ \\
\hline Helianthus decapetalus L. & Asteraceae & $\mathrm{P}$ & $?$ \\
\hline Helianthus $\times$ laetiflorus Pers. & Asteraceae & $\mathrm{P}$ & $?$ \\
\hline Helleborus viridis L. & Ranunculaceae & $\mathrm{P}$ & $4 a$ \\
\hline Hemerocallis fulva (L.) L. & Liliaceae & $\mathrm{P}$ & $4 b$ \\
\hline Hesperis sylvestris Crantz & Brassicaceae & $\mathrm{BP}$ & $4 a$ \\
\hline Hibiscus trionum L. & Malvaceae & A & $2 \mathrm{a}$ \\
\hline Hieracium aurantiacum L. ${ }^{15}$ & Asteraceae & $\mathrm{P}$ & $2 \mathrm{a}, 4 \mathrm{a}$ \\
\hline Hordeum jubatum L. & Poaceae & $\mathrm{P}$ & $4 a$ \\
\hline Hordeum vulgare L. em. Alef. ${ }^{16}$ & Poaceae & $\mathrm{A}$ & $4 \mathrm{~b}$ \\
\hline Hyssopus officinalis L. & Lamiaceae & $\mathrm{S}$ & $4 a$ \\
\hline Iberis amara L. & Brassicaceae & $\mathrm{A}$ & $4 a$ \\
\hline Iberis umbellata L. & Brassicaceae & A & $4 \mathrm{~b}$ \\
\hline Impatiens balsamina L. & Balsaminaceae & A & $4 a$ \\
\hline $\begin{array}{l}\text { Impatiens stenosepala E. Pritz. } \\
\text { [= Impatiens punctata Franch. ex Hook. f.] }\end{array}$ & Balsaminaceae & A & $4 a$ \\
\hline Ipomoea purpurea Roth & Convolvulaceae & $\mathrm{A}$ & $4 a$ \\
\hline $\begin{array}{l}\text { Ipomoea nil (L.) Roth } \\
\text { [= Ipomoea hederacea (L.) Jacq.] }\end{array}$ & Convolvulaceae & A & $4 a$ \\
\hline Iris germanica L. & Iridaceae & $\mathrm{P}$ & $4 \mathrm{~b}$ \\
\hline Isatis tinctoria L. & Brassicaceae & $\mathrm{BP}$ & $4 a$ \\
\hline
\end{tabular}

In Poland, it is a native species in the Carpathians (Zając \& Zając 2001). In lowlands, it appears only ephemerally (Szulczewski 1951)

In Poland, it is a native species in the Tatras and Pieniny Mts (Zając \& Zając 2001). In lowlands, this species occurs only as an escapee from cultivation.

In Poland, it is a native species in the mountains and in the foothills (Zając \& Zając 2001). In lowlands, this species occurs only as an escapee from cultivation.

16 Hordeum distichon L., a species reported from Wielkopolska has been included here in Hordeum vulgare L. em. Alef. 


\begin{tabular}{|c|c|c|c|}
\hline Name of species & Name of family & BF & OP \\
\hline Larix $\times$ eurolepis A. Henry ${ }^{17}$ & Pinaceae & $\mathrm{T}$ & $4 b$ \\
\hline Lathyrus hirsutus L. ${ }^{18}$ & Fabaceae & A & $4 a ?$ \\
\hline $\begin{array}{l}\text { Lathyrus japonicus Willd. subsp. maritimus } \\
\text { (L.) P. W. Ball }{ }^{19}\end{array}$ & Fabaceae & $\mathrm{P}$ & $2 \mathrm{a}$ \\
\hline Lathyrus latifolius L. & Fabaceae & $\mathrm{P}$ & $4 \mathrm{a}$ \\
\hline Lathyrus nissolia L. & Fabaceae & A & $?$ \\
\hline Lavandula angustifolia Mill. & Lamiaceae & $\mathrm{S}$ & $4 \mathrm{a}$ \\
\hline Lavatera trimestris $\mathrm{L}$. & Malvaceae & A & $4 a$ \\
\hline Lens culinaris Medik. & Fabaceae & A & $2 \mathrm{a}$ \\
\hline Lepidium sativum L. & Brassicaceae & A & $4 \mathrm{~b}$ \\
\hline Leucanthemum maximum (Ramond) DC. & Asteraceae & $\mathrm{P}$ & $4 a$ \\
\hline Levisticum officinale W. D. J. Koch & Apiaceae & $\mathrm{P}$ & $4 \mathrm{~b}$ \\
\hline Lilium bulbiferum L. ${ }^{20}$ & Liliaceae & $\mathrm{P}$ & $4 \mathrm{~b}$ \\
\hline Lilium candidum L. & Liliaceae & $\mathrm{P}$ & $4 \mathrm{a}$ \\
\hline Linum grandiflorum Desf. & Linaceae & A & $4 a$ \\
\hline Linum perenne L. & Linaceae & $\mathrm{P}$ & $2 \mathrm{a}$ \\
\hline Linum usitatissimum L. & Linaceae & A & $4 \mathrm{~b}$ \\
\hline Lobelia erinus L. & Campanulaceae & A & $?$ \\
\hline Lobularia maritima (L.) Desv. & Brassicaceae & AP & $4 a$ \\
\hline Lonicera caprifolium L. & Caprifoliaceae & $\mathrm{T}$ & $4 \mathrm{a}$ \\
\hline $\begin{array}{l}\text { Lonicera involucrata (Richardson) Banks ex } \\
\text { Spreng. }\end{array}$ & Caprifoliaceae & $\mathrm{T}$ & $4 \mathrm{a}$ \\
\hline Lunaria annua L. & Brassicaceae & $\mathrm{AB}$ & $4 b$ \\
\hline Lupinus angustifolius L. & Fabaceae & A & $4 \mathrm{a}$ \\
\hline Lupinus luteus L. & Fabaceae & A & $4 \mathrm{~b}$ \\
\hline Lychnis coronaria (L.) Desr. & Caryophyllaceae & $\mathrm{P}$ & $4 \mathrm{~b}$ \\
\hline Lysimachia punctata L. & Primulaceae & $\mathrm{P}$ & $4 a$ \\
\hline Malva verticillata $L$. & Malvaceae & A & $4 \mathrm{~b}$ \\
\hline Mathiola incana (L.) W. T. Aiton & Brassicaceae & A & $4 a ?$ \\
\hline Melica altissima L. & Poaceae & $\mathrm{P}$ & $?$ \\
\hline Melica ciliata L. ${ }^{21}$ & Poaceae & $\mathrm{P}$ & $?$ \\
\hline Melissa officinalis L. & Lamiaceae & $\mathrm{P}$ & $4 a$ \\
\hline $\begin{array}{l}\text { Mentha } \times \text { piperita L. } \\
{[=\text { Mentha } \times \text { citrata Ehrh. }]}\end{array}$ & Lamiaceae & $\mathrm{P}$ & $4 \mathrm{a}$ \\
\hline Mentha $\times$ rotundifolia (L.) Huds. & Lamiaceae & $\mathrm{P}$ & $4 a$ \\
\hline Mentha spicata L. em. L. & Lamiaceae & $\mathrm{P}$ & $2 \mathrm{a}$ \\
\hline Miscanthus sacchariflorus (Maxim.) Hack. & Poaceae & $\mathrm{B}$ & $4 \mathrm{~b}$ \\
\hline Morus alba L. & Moraceae & $\mathrm{T}$ & $4 b$ \\
\hline Muscari botryoides (L.) Mill. & Liliaceae & $\mathrm{P}$ & $4 a$ \\
\hline Muscari comosum (L.) Mill. ${ }^{22}$ & Liliaceae & $\mathrm{P}$ & $2 \mathrm{a}, 4 \mathrm{a}$ \\
\hline Muscari neglectum Guss. ex Ten. & Liliaceae & $\mathrm{P}$ & $4 a$ \\
\hline Myrrhis odorata (L.) Scop. & Apiaceae & $\mathrm{P}$ & $?$ \\
\hline Narcissus poeticus L. & Amaryllidaceae & $\mathrm{P}$ & $4 \mathrm{~b}$ \\
\hline Narcissus pseudonarcissus L. & Amaryllidaceae & $\mathrm{P}$ & $4 b$ \\
\hline Nepeta grandiflora M. Bieb. & Lamiaceae & $\mathrm{P}$ & $2 \mathrm{a}$ \\
\hline $\begin{array}{l}\text { Nepeta racemosa Lam. } \\
\text { [= Nepeta mussinii Spreng. ex Henckel }]\end{array}$ & Lamiaceae & $\mathrm{P}$ & $2 \mathrm{a}$ \\
\hline
\end{tabular}

Hybrid of Larix decidua Mill. subsp. decidua and L. kaempferi (Lamb.) Carrière. It is cultivated and running wild from cultivation (Danielewicz \& Maliński 1994, 1997; Purcel 2009).

18 Only some localities in southern Poland are native (Zając \& Zając 2001).

19 Only some localities on the Baltic Sea are native in Poland (Zając \& Zając 2001). The species was reported from Poznań by Szulczewski (1951).

20 Only certain mountain localities are native in Poland (Zając \& Zając 2001).

21 Only the localities in the south-eastern part of the country are native in Poland (Zając \& Zając 2001). In Wielkopolska: Trzemeszno surroundings (Pampuch 1840; Ritschl 1850).

22 Native in southern Poland (Zając \& Zając 2001). In Wielkopolska, this species runs wild from cultivation. 


\begin{tabular}{|c|c|c|c|}
\hline Name of species & Name of family & BF & OP \\
\hline Nicandra physalodes (L.) Gaertn. & Solanaceae & A & $4 a$ \\
\hline Nigella damascena L. & Ranunculaceae & A & $4 \mathrm{~b}$ \\
\hline $\begin{array}{l}\text { Nonea erecta Bernh. } .^{23} \\
{[=\text { Nonea pulla (L.) DC. }]}\end{array}$ & Boraginaceae & $\mathrm{P}$ & $2 \mathrm{a}$ \\
\hline Oenothera fruticosa L. & Onagraceae & $\mathrm{P}$ & $4 a$ \\
\hline Oenothera glazioviana Micheli & Onagraceae & $\mathrm{B}$ & $4 a$ \\
\hline Omphalodes verna Moench & Boraginaceae & $\mathrm{P}$ & $4 \mathrm{~b}$ \\
\hline Ornithogalum nutans L. & Liliaceae & $\mathrm{P}$ & $4 \mathrm{~b}$ \\
\hline Ornithopus sativus Brot. & Fabaceae & A & $4 \mathrm{~b}$ \\
\hline Panicum miliaceum L. & Poaceae & A & $4 \mathrm{~b}$ \\
\hline Papaver orientale L. & Papaveraceae & $\mathrm{P}$ & $4 a$ \\
\hline Papaver somniferum L. & Papaveraceae & A & $4 \mathrm{~b}$ \\
\hline Petroselinum crispum (Mill.) A. W. Hill & Apiaceae & $\mathrm{B}$ & $4 \mathrm{a}$ \\
\hline Petunia $\times$ hybrida (Hook.) Vilm. & Solanaceae & A & $4 a$ \\
\hline $\begin{array}{l}\text { Petunia integrifolia (Hook.) Schinz et Thell. } \\
\text { [= Petunia violacea Lindl. }]\end{array}$ & Solanaceae & A & $2 \mathrm{a}, 4 \mathrm{a}$ \\
\hline Peucedanum ostruthium (L.) W. D. J. Koch ${ }^{24}$ & Apiaceae & $\mathrm{P}$ & $3 a ?$ \\
\hline Phacelia tanacaetifolia Benth. & Hydrophyllaceae & A & $4 \mathrm{~b}$ \\
\hline Phaseolus vulgaris L. & Fabaceae & A & $4 \mathrm{a}$ \\
\hline Philadelphus coronarius L. & Hydrangeaceae & $\mathrm{T}$ & $4 \mathrm{~b}$ \\
\hline Physalis alkekengi L. & Solanaceae & $\mathrm{P}$ & $4 \mathrm{~b}$ \\
\hline Physalis ixocarpa Brot. ex Hornem. & Solanaceae & A & $?$ \\
\hline Physocarpus opulifolius (L.) Maxim. & Rosaceae & $\mathrm{T}$ & $4 \mathrm{~b}$ \\
\hline Phyteuma nigrum F. W. Schmidt & Campanulaceae & $\mathrm{P}$ & $?$ \\
\hline Phytolacca americana L. ${ }^{25}$ & Phytolaccaceae & $\mathrm{P}$ & $2 \mathrm{a}$ \\
\hline $\begin{array}{l}\text { Phytolacca esculenta van Houtte }{ }^{26} \\
\text { [= Phytolacca acinosa Roxb.] }\end{array}$ & Phytolaccaceae & $\mathrm{P}$ & $4 a$ \\
\hline Pimpinella anisum $\mathrm{L}$. & Apiaceae & A & $?$ \\
\hline Pinus banksiana Lamb. & Pinaceae & $\mathrm{T}$ & $4 \mathrm{a}$ \\
\hline Pinus nigra J. F. Arnold & Pinaceae & $\mathrm{T}$ & $4 \mathrm{a}$ \\
\hline Pinus rigida Mill. & Pinaceae & $\mathrm{T}$ & $4 \mathrm{~b}$ \\
\hline Pinus strobus L. & Pinaceae & $\mathrm{T}$ & $4 \mathrm{~b}$ \\
\hline Pisum sativum L. & Fabaceae & A & $4 \mathrm{~b}$ \\
\hline Plantago coronopus $\mathrm{L}^{27}$ & Plantaginaceae & ABP & $4 \mathrm{~b}$ \\
\hline Populus balsamifera L. ${ }^{28}$ & Salicaceae & $\mathrm{T}$ & $4 \mathrm{a}$ \\
\hline $\begin{array}{l}\text { Populus suaveolens Fisch. ex Loudon }{ }^{29} \\
\text { [= Populus maximowiczii Henry }]\end{array}$ & Salicaceae & $\mathrm{T}$ & $4 \mathrm{a}$ \\
\hline Prunus domestica L. ${ }^{30}$ & Rosaceae & $\mathrm{T}$ & $4 \mathrm{~b}$ \\
\hline $\begin{array}{l}\text { Prunus persica }(\mathrm{L} .) \text { Batsch } \\
\text { [= Persica vulgaris Mill. }]\end{array}$ & Rosaceae & $\mathrm{T}$ & $4 \mathrm{~b}$ \\
\hline $\begin{array}{l}\text { Prunus armeniaca L. } \\
\text { [= Armeniaca vulgaris Lam.] }\end{array}$ & Rosaceae & $\mathrm{T}$ & $4 b$ \\
\hline $\begin{array}{l}\text { Prunus cerasus L. } \\
\text { [= Cerasus vulgaris Mill.] }\end{array}$ & Rosaceae & $\mathrm{T}$ & $4 b$ \\
\hline
\end{tabular}

23 Native in the south-eastern part of Poland (Zając \& Zając 2001).

24 In Poland, native localities are located in the Sudetes (Zając \& Zając 2001). In Wielkopolska, this species is running wild from cultivation.

25 Reported from Gubin (Lademann 1937).

26 Reported from the Wielkopolski National Park (Latowski 2009).

27 The only native locality in Poland is on Kępa Karsiborska (Sotek 2014). The species was noted at the roadside near the Botanical Garden in Poznan. Perhaps, this population originated from the conservative collection of this species located in the Botanical Garden.

28 In Wielkopolska, the balsam poplar section (Tacamahaca) is represented by, among others: Populus $\times$ berolinensis (K. Koch) Dippel, P. candicans Aiton and P. simonii Carrière.

29 Populus suaveolens Fisch. ex Loudon (P. maximowiczii Henry) has some varieties, e.g., 'NE42' (= 'Hybrida 275'), which reproduce vegetatively by suckers, e.g., in excavations (Chmiel 1993, 2006; Dolatowski \& Seneta 2008).

30 The species is diversified into several subspecies with varying degrees of spread and establishment in the region, such as, among others: subsp. domestica; subsp. insititia (L.) Bonnier et Layens and subsp. syriaca (Borkh.) Janch. ex Mansf. 


\begin{tabular}{|c|c|c|c|}
\hline Name of species & Name of family & BF & OP \\
\hline Pseudotsuga menziesii (Mirbel) Franco & Pinaceae & $\mathrm{T}$ & $4 a$ \\
\hline Ptelea trifoliata L. & Rutaceae & $\mathrm{T}$ & $4 a$ \\
\hline Pyrus communis L. ${ }^{31}$ & Rosaceae & $\mathrm{T}$ & $4 b$ \\
\hline Raphanus sativus L. & Brassicaceae & A & $4 b$ \\
\hline Reseda odorata L. & Resedaceae & A & $4 a$ \\
\hline Reseda phyteuma L. ${ }^{32}$ & Resedaceae & A & $2 \mathrm{a}$ \\
\hline Rheum rhabarbarum L. & Polygonaceae & $\mathrm{P}$ & $4 b$ \\
\hline $\begin{array}{l}\text { Rhus hirta (L.) Sudw. } \\
\text { [= Rhus typhina L.] }\end{array}$ & Anacardiaceae & $\mathrm{T}$ & $4 b$ \\
\hline Ribes aureum Pursh & Grossulariaceae & $\mathrm{T}$ & $4 b$ \\
\hline Ribes rubrum L. & Grossulariaceae & $\mathrm{T}$ & $4 b$ \\
\hline Robinia neomexicana A. Gray ${ }^{33}$ & Fabaceae & $\mathrm{T}$ & $4 a$ \\
\hline Robinia viscosa Vent. ${ }^{34}$ & Fabaceae & $\mathrm{T}$ & $4 a$ \\
\hline Rosa blanda Aiton & Rosaceae & $\mathrm{T}$ & $4 \mathrm{a}$ \\
\hline Rosa glauca Pourr. & Rosaceae & $\mathrm{T}$ & $4 a$ \\
\hline Rosa multiflora Thunb. ex Murray & Rosaceae & $\mathrm{T}$ & $4 a$ \\
\hline Rosa rugosa Thunb. & Rosaceae & $\mathrm{T}$ & $4 b$ \\
\hline Rosa spinosissima L. & Rosaceae & $\mathrm{T}$ & $4 \mathrm{a}$ \\
\hline Rosa virginiana Herrm. & Rosaceae & $\mathrm{T}$ & $4 a$ \\
\hline Rubus occidentalis L. & Rosaceae & $\mathrm{T}$ & $4 a$ \\
\hline Rudbeckia hirta L. & Asteraceae & $\mathrm{ABP}$ & $4 b$ \\
\hline Salix acutifolia Willd. & Salicaceae & $\mathrm{T}$ & $4 \mathrm{a}$ \\
\hline Salix cordata Michx & Salicaceae & $\mathrm{T}$ & $4 a$ \\
\hline Salix daphnoides Vill. ${ }^{35}$ & Salicaceae & $\mathrm{T}$ & $?$ \\
\hline Salix eleagnos Scop. ${ }^{36}$ & Salicaceae & $\mathrm{T}$ & $?$ \\
\hline Salvia glutinosa L. ${ }^{37}$ & Lamiaceae & $\mathrm{P}$ & $4 a$ \\
\hline Sambucus ebulus L. ${ }^{38}$ & Caprifoliaceae & $\mathrm{P}$ & $4 a$ \\
\hline Satureja hortensis L. & Lamiaceae & A & $?$ \\
\hline Scilla bifolia L. ${ }^{39}$ & Liliaceae & $\mathrm{P}$ & $2 \mathrm{a}, 4 \mathrm{a}$ \\
\hline Scilla sibirica Haw. & Liliaceae & $\mathrm{P}$ & $4 b$ \\
\hline Scorzonera hispanica L. & Asteraceae & $\mathrm{P}$ & $2 \mathrm{a}$ \\
\hline Scrophularia vernalis L. ${ }^{40}$ & Scrophulariaceae & $\mathrm{BP}$ & $4 \mathrm{a}$ \\
\hline Secale cereale L. ${ }^{41}$ & Poaceae & A & $4 a$ \\
\hline Sempervivum tectorum L. & Crassulaceae & $\mathrm{P}$ & $4 b$ \\
\hline Setaria italica (L.) P. Beauv. & Poaceae & A & $4 a$ \\
\hline Sicyos angulatus L. & Cucurbitaceae & $\mathrm{A}$ & $4 \mathrm{a}$ \\
\hline Sida hermaphrodita (L.) Rusby & Malvaceae & $\mathrm{P}$ & $4 a$ \\
\hline Silene armeria L. ${ }^{42}$ & Caryophyllaceae & AP & $2 \mathrm{a}$ \\
\hline $\begin{array}{l}\text { Silene chalcedonica (L.) E. Krause } \\
\text { [= Lychnis chalcedonica L.] }\end{array}$ & Caryophyllaceae & $\mathrm{P}$ & $4 \mathrm{a}$ \\
\hline
\end{tabular}

31 Very variable and often hybridizing species, e.g., P. $\times$ myloslavensis A. Czarna \& W. Antkowiak $=$ P. communis L. $\times$ P. salicifolia Pall. (Antkowiak et al. 2008). The checklist does not include Mespilus germanica L. (= Pyrus germanica Hook. F.), a species previously reported by Szulczewski (1951).

32 Only localities in the Nidziańska Basin are native in Poland (Kaźmierczakowa 2014b). The species was reported from Łopuchowo near Oborniki (Wielkopolska) by Szulczewski (1951).

33 Robinia neomexicana A. Gray hybridizes with R. pseudoacacia L. $=R . \times$ holdtii Beissn. (Zieliński et al. 2015; Zieliński 2016).

34 Robinia viscosa Vent. hybridizes with $R$. pseudoacacia L. = R. $\times$ ambigua Poir. (see Zielinski et al. 2015, Zieliński 2016).

35 A native species on the coast and in the mountains (Zając \& Zajac 2001), besides, planted and running wild in lowlands.

36 Native in the mountains and in the foothills (Zając \& Zając 2001), besides, planted and running wild in lowlands.

37 Native in southern Poland (Zając \& Zając 2001). In addition, it was reported from Wielkopolska: Poznań (Czarna \& Mielcarski 2004) and in the vicinity of Konin (leg. et det. J. Chmiel 2011, POZ).

38 Native in southern Poland (Zając \& Zając 2001); in Wielkopolska, running wild from cultivation.

39 Native in south-eastern Poland and the Upper Silesia (Zając \& Zając 2001); in Wielkopolska, running wild from cultivation (Szulczewski 1951).

40 Running wild from cultivation in the Botanical Garden in Poznań (W. Rakowski, P. Górski, D. Tomaszewski, W. Stachnowicz, pers. comm., 1992).

41 In Wielkopolska, an intergeneric wild hybrid also occurs $\times$ Triticosecale blaringhemii A. Camus $=\times$ Triticale rimpaui Wittm. (Secale cereale $\mathrm{L} . \times$ Triticum aestivum L. em. Fiori et Paol.), which is often cultivated.

42 Szulczewski (1951) also reports Silene nemoralis Waldst et Kit. [Silene italica subsp. nemoralis (Waldst. et Kit.) Nyman] from Wielkopolska, which is a native species in the mountains. 


\begin{tabular}{|c|c|c|c|}
\hline Name of species & Name of family & BF & OP \\
\hline Silybum marianum (L.) Gaertn. & Asteraceae & $\mathrm{AB}$ & $4 \mathrm{~b}$ \\
\hline Sinapis alba L. & Brassicaceae & A & $4 \mathrm{~b}$ \\
\hline Solanum tuberosum L. & Solanaceae & $\mathrm{P}$ & $4 a$ \\
\hline Solidago graminifolia (L.) Salisb. & Asteraceae & $\mathrm{P}$ & $4 \mathrm{~b}$ \\
\hline Solidago patula Muhl. ex Willd. ${ }^{43}$ & Asteraceae & $\mathrm{P}$ & $2 \mathrm{a}$ \\
\hline Sorbaria kirilowii (Regel \& Tiling) Maxim. & Rosaceae & $\mathrm{T}$ & $4 \mathrm{a}$ \\
\hline Sorbaria sorbifolia (L.) A. Braun & Rosaceae & $\mathrm{T}$ & $4 a$ \\
\hline Sorbus aria (L.) Crantz s. str. & Rosaceae & $\mathrm{T}$ & $4 a$ \\
\hline Sorbus hybrida L. ${ }^{44}$ & Rosaceae & $\mathrm{T}$ & $4 a$ \\
\hline Sorbus intermedia (Ehrh.) Pers. ${ }^{45}$ & Rosaceae & $\mathrm{T}$ & $4 \mathrm{~b}$ \\
\hline Spinacia oleracea L. & Chenopodiaceae & A & $2 a$ \\
\hline Spiraea $\times$ biilardii Herincq & Rosaceae & $\mathrm{T}$ & $4 \mathrm{a}$ \\
\hline Spiraea $\times$ pseudosalicifolia Silverside ${ }^{46}$ & Rosaceae & $\mathrm{T}$ & $4 \mathrm{a}$ \\
\hline Spiraea ×vanhouttei (Briot) Zabel & Rosaceae & $\mathrm{T}$ & $4 \mathrm{a}$ \\
\hline Spiraea chamaedryfolia L. & Rosaceae & $\mathrm{T}$ & $4 \mathrm{~b}$ \\
\hline Spiraea media Schmidt & Rosaceae & $\mathrm{T}$ & $4 \mathrm{~b}$ \\
\hline Spiraea salicifolia L. ${ }^{47}$ & Rosaceae & $\mathrm{T}$ & $4 \mathrm{a}$ \\
\hline Stachys byzantina K. Koch & Lamiaceae & $\mathrm{P}$ & $4 \mathrm{a}$ \\
\hline Staphylea pinnata L. ${ }^{48}$ & Staphyleaceae & $\mathrm{T}$ & $2 \mathrm{a}$ \\
\hline Symphytum cordatum Waldst. et Kit. ${ }^{49}$ & Boraginaceae & $\mathrm{P}$ & $2 \mathrm{a}$ \\
\hline Syringa vulgaris $\mathrm{L}$. & Oleaceae & $\mathrm{T}$ & $4 \mathrm{a}$ \\
\hline Tagetes erecta L. & Asteraceae & A & $4 \mathrm{a}$ \\
\hline Tagetes tenuifolia Cav. & Asteraceae & A & $4 \mathrm{a}$ \\
\hline Tanacetum balsamita L. & Asteraceae & $\mathrm{P}$ & $4 \mathrm{a}$ \\
\hline Telekia speciosa (Schreb.) Baumg. & Asteraceae & $\mathrm{P}$ & $4 \mathrm{a}$ \\
\hline Thuja occidentalis L. & Cupressaceae & $\mathrm{T}$ & $4 \mathrm{~b}$ \\
\hline Thuja plicata Donn ex D. Don & Cupressaceae & $\mathrm{T}$ & $4 \mathrm{~b}$ \\
\hline Thymus vulgaris L. & Lamiaceae & S & $4 \mathrm{a}$ \\
\hline Tragopogon porrifolius L. & Asteraceae & $\mathrm{ABP}$ & $2 \mathrm{a}$ \\
\hline Trifolium incarnatum L. & Fabaceae & A & $4 \mathrm{~b}$ \\
\hline Trifolium resupinatum L. & Fabaceae & A & $4 \mathrm{~b}$ \\
\hline Trigonella coerulea (L.) Ser. & Fabaceae & A & $2 \mathrm{a}$ \\
\hline $\begin{array}{l}\text { Triticum aestivum L. em. Fiori et Paol. }{ }^{50} \\
\text { [= Triticum vulgare Vill. }]\end{array}$ & Poaceae & A & $4 \mathrm{~b}$ \\
\hline Triticum spelta L. & Poaceae & A & $?$ \\
\hline Tropaeolum majus L. & Tropaeolaceae & A & $4 \mathrm{~b}$ \\
\hline Tulipa gesneriana L. & Liliaceae & $\mathrm{P}$ & $4 \mathrm{~b}$ \\
\hline Tulipa sylvestris L. & Liliaceae & $\mathrm{P}$ & $2 \mathrm{a}$ \\
\hline Tulipa tarda Stapf & Liliaceae & $\mathrm{P}$ & $4 a$ \\
\hline Typha laxmanii Lepech. ${ }^{51}$ & Typhaceae & $\mathrm{P}$ & $4 \mathrm{~b}$ \\
\hline Typha minima Hoppe & Typhaceae & $P$ & $?$ \\
\hline Verbena rigida Spreng. & Verbenaceae & A & $3 a ?$ \\
\hline Verbena urticifolia L. & Verbenaceae & $\mathrm{ABP}$ & $3 a$ ? \\
\hline Viburnum lantana L. & Caprifoliaceae & $\mathrm{T}$ & $4 \mathrm{a}$ \\
\hline Vicia faba $L$. & Fabaceae & A & $4 \mathrm{a}$ \\
\hline
\end{tabular}

43 Reported from Gniezno (Szulczewski 1951).

44 Reported from the Wielkopolski National Park (Purcel 2009).

In Wielkopolska, the species is planted and running wild from cultivation.

Sometimes confused with Spiraea $\times$ billardii auct. non Hérincq (Purcel 2007).

47 Native only in the south-eastern part of Poland (Zając \& Zając 2001); in Wielkopolska, running wild from cultivation.

48 Native only in the south part of Poland (Zajac \& Zajac 2001); in Wielkopolska, running wild from cultivation (Szulczewski 1951).

9 Native only in the south-eastern part of Poland (Zając \& Zając 2001); in Wielkopolska, running wild from cultivation (Szulczewski 1951).

See note 41 .

Specimens of this species were collected near Konin (leg. and det. J. Chmiel 2008, POZ) and Barcin (leg. et det. J. Chmiel 2016, POZ). 


\begin{tabular}{llcc}
\hline Name of species & Name of family & BF & OP \\
\hline Vicia sativa L. & Fabaceae & A & $4 \mathrm{~b}$ \\
Vinca minor L. ${ }^{52}$ & Apocynaceae & $\mathrm{S}$ & $4 \mathrm{~b}$ \\
Viola suavis M. Bieb. & Violaceae & $\mathrm{P}$ & $3 \mathrm{a} ?$ \\
Viola wittrockiana Gams & Violaceae & $\mathrm{AP}$ & $4 \mathrm{~b}$ \\
Vitis vinifera L. & Vitaceae & $\mathrm{T}$ & $4 \mathrm{~b}$ \\
\hline
\end{tabular}

Explanations: BF - biological form, A - annual plants, B - biennial plants, $\mathrm{P}$ - perennial, herbaceous plants, $\mathrm{S}$ - dwarf shrubs and sub-shrubs, $\mathrm{T}$ - shrubs and trees; OP - observation period, 1 - to 1901, 2 - 1901-1951, 3 - 1951-2001, 4 - after 2001, a - species was observed once, b - species was observed repeatedly; ? - no data information uncertain 